hep-th/0306030

SU-ITP-03/11, SLAC-PUB-9907

\title{
On Branes and Oriented B-fields
}

\author{
Keshav Dasgupta and Marina Shmakova ${ }^{2}$ \\ ${ }^{1}$ Varian Laboratory of Physics, Stanford University, Stanford CA 94305-4060, USA \\ ${ }^{2}$ Stanford Linear Accelerator Center, Stanford University, Stanford CA 94309, USA
}

\begin{abstract}
Novel theories appear on the world-volume of branes by orienting $B$ fields along various directions of the branes. We review some of the earlier developments and explore many new examples of these theories. In particular, among other things, we study the pinning effect of branes near conifold like singularities and brane-antibrane theories with different fluxes on their world-volumes. We show that all these theories arise from different limits of an M-theory configuration with appropriately chosen G-fluxes. This gives us a way to study them from a unified framework in M-theory.
\end{abstract}

Submitted to Physical Review D

May 2003

1 keshav@itp.stanford.edu

2 shmakova@slac.stanford.edu 


\section{Contents}

1. Introduction . . . . . . . . . . . . . . . . . . . . . . . . . . . . . . . . . 1

2. Theory Near Single-Centered Taub-NUT Geometry . . . . . . . . . . . . . . . . 3

2.1. Pinned Branes . . . . . . . . . . . . . . . . . . . . . . . . . . . . . . . 1

2.2. Dipole Theories . . . . . . . . . . . . . . . . . . . . . . . . . . . . . . 5

2.3. Noncommutative Geometry . . . . . . . . . . . . . . . . . . . . . . . . . 7

3. Theory Near Conifold Type Geometry . . . . . . . . . . . . . . . . . . . . . . 10

3.1. Pinned Branes . . . . . . . . . . . . . . . . . . . . . . . . . . . . . . . 11

3.2. Hybrid Theory: Noncommutative Geometry and Dipole Theory . . . . . . . . . 13

4. Theory Near Multi-Centered Taub-NUT Geometry . . . . . . . . . . . . . . . . . 15

4.1. Pinned Branes And Dipole Theory . . . . . . . . . . . . . . . . . . . . . . 16

4.2. Noncommutative Geometry . . . . . . . . . . . . . . . . . . . . . . . . . 17

4.3. Nonabelian Geometry . . . . . . . . . . . . . . . . . . . . . . . . . . . . 21

5. Some Applications . . . . . . . . . . . . . . . . . . . . . . . . . . . . . . . 23

5.1. Parallel Branes . . . . . . . . . . . . . . . . . . . . . . . . . . . . . . . 24

5.2. Brane-Antibrane theory . . . . . . . . . . . . . . . . . . . . . . . . . . . 26

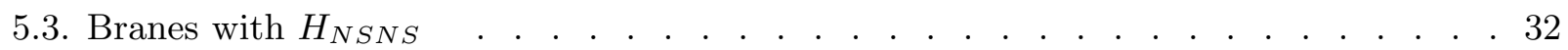

5.4. Theory near multi-Conifold type geometry . . . . . . . . . . . . . . . . . . . 35

6. Discussions . . . . . . . . . . . . . . . . . . . . . . . . . . . . . . . . . . 37

\section{Introduction}

Some time back theories on a M5 brane with lower dimensional poincare invariance were considered. These theories suggested a way to localise branes in ambient space. To generalise this further we consider two cases. In one case, which was studied earlier in some detail [1], we keep a M5 brane near a single centred Taub-NUT space. When a Lorentz noninvariant tensor background is switched on we get a $(1,0)$ theory with a massive hypermultiplet in six dimensions. This theory actually has a $4+1$ dimensional Poincare symmetry. In the other case we keep the M5 brane near a conifold like singularity. When a tensor background is switched on, we get a theory with only $3+1$ dimensional poincare invariance. However, as we shall see, this lowered poincare invariance is a consequence of the geometry of the problem and not of the background tensor field.

The above discussion is only a small part of a more unified story that we want to present here. It was studied earlier that switching on anti-symmetric two form fluxes the usual $B_{N S}$ fluxes of string theory - on the brane results in making the coordinates non-commutative. This gave rise to the non-commutative geometry as being an embedding of string theory. The noncommutative geometry was elaborated in subsequent papers and many interesting details were shown to occur. It was shown that the dynamics are governed 
by a star product. In terms of the star product, both the fields and the coordinates are noncommutative.

The noncommutative theory is also nonlocal in nature. However this is more involved. There do exist a slightly simpler setup where the noncommutativity only extends to the fields and not to the coordinates. The coordinates remain commutative. This theory is also nonlocal by construction and is called the Dipole theory, first constructed by Bergman and Ganor [2]. Whereas the noncommutative theory can have a maximal supersymmetry of $\mathcal{N}=4$, the dipole theory on the other hand can have a maximal susy of $\mathcal{N}=2$. Both the dipole and the noncommutative theory have supergravity duals whose boundary metric degenerates along some of the directions. These details have been explicitly shown in [3] for the noncommutative geometry and in [4] for the dipole theory. Since the coordinates commute, the star product for the dipole theory is given by

$$
\Phi(x) \star \Psi(x)=\left.e^{\frac{1}{2}\left(L_{1} \cdot \frac{\partial}{\partial y}-L_{2} \cdot \frac{\partial}{\partial w}\right)} \Phi(w) \Psi(y)\right|^{y=w=x}
$$

where $L_{1,2}$ are the dipole lengths of the fields $\Phi$ and $\Psi$ respectively. This star product is associative. As been discussed in [5] the dipole theory is derived from $\mathcal{N}=4 \mathrm{SYM}$ theory by the inclusion of a dimension five operator. This is again simpler than noncommutative theory, where we need a dimension six operator.

An immediate extension of the above scenario is to consider the non-abelian case by putting many branes parallel to each other. There are two different scenarios now: (a) We have same $B$ fields on all the parallel branes, and (b) Different branes have different, but constant, $B$ fields. The first case is just a simple extension of the Moyal star product in which the non-abelian nature do not mix in any way with the noncommutativity. The second case is more interesting as been shown in [6]. One could in fact evaluate the star product which mixes both the non-abelian nature and the noncommutativity of the branes in a unified way to give the following nonabelian product:

$$
(\Phi \times \Psi)_{k}^{i}(X) \equiv \sum_{j}\left(\Phi_{j}^{i} \star_{i j k} \Psi_{k}^{j}\right)(X),
$$

where $i, j, k$ label the branes and $\star_{i j k}$ depends on all three indices. This star product is associative and the derivation of this has been given in details in [6]. In this paper we will elaborate some more properties of this star product and discuss a situation where there is a stable configuration.

All these configurations discussed above and in earlier papers give us a setup which we call branes with oriented $B$ fields. Imagine a brane located at the degenerating point of a 
Taub-NUT space with a $B$ flux switched on. The Taub-NUT is required to have non-trivial configuration of $B$ fields so that we cannot gauge it away. Following four interesting cases arise now:

(a) $B$ field is completely orthogonal to the brane. This is the pinned brane scenario where the brane is pinned at the origin of the Taub-NUT. The hypermultiplets in this theory become massive and therefore the susy is reduced [1].

(b) $B$ field has one leg along the brane and the other leg perpendicular to it. This is the dipole theory. The brane is no longer pinned but now the hypers on the world volume develop a constant dipole length. The vectors still have zero dipole length [2, 5, 4, (7].

(c) $B$ field has both the legs on the brane. This is the noncommutative geometry [8,9,10]. The theory also develop dipoles but the dipole lengths are not constant rather they depend on the momentum [11,12].

(d) $B$ fields have different (but constant) values on parallel branes. This is the nonabelian geometry. The theory mixes both the noncommutativity and the nonabelian nature in a single star product [6].

All these theories have been studied earlier. Some in great details and some in not so great details. However all these theories, though look different, do actually arise from a single configuration in M-theory which we shall elaborate in details here. We shall review some of these earlier works, now in the light of the unified picture, and provide many new examples of these theories. The M-theory framework which unifies the set of theory described above is simply a background with a M5 brane probing a Taub-NUT with G-fluxes. We shall discuss this in section 2.

But there is more. The four dimensional Taub-NUT background (which is probed by branes) could be fibered non-trivially over a $P^{1}$ base. In fact this background is related to a six dimensional conifold type geometry with fluxes! The M5 probing this background has now new set of theories which we shall elaborate in section 3 .

We could even extend this further by making the Taub-NUT geometry multi- centered. In the presence of various different choices of localised G-fluxes, the nonabelian geometry could be described. We will show in section 4 how various properties of these geometries arise from a multi Taub-NUT background.

In section 5 some applications of the various scenarios will be presented. We will also point out some possibilities of getting new theories that are somewhat orthogonal (though related) to the material presented in the earlier sections. In fact we will argue that the multi Taub-NUT geometry studied in section 4 can now be fibered over a $P^{1}$ (as in section 2 ) to get a multi-conifold like geometry; and therefore will give a set of new theories. We will present our conclusions in section 6 and show that even time dependent backgrounds can be tackled using the generic scheme presented in the earlier sections. 


\section{Theory Near Single-Centered Taub-NUT Geometry}

What we require is the metric of a Taub-NUT space in M-theory and in the presence of G-fluxes when we allow maximal possible rank for the fluxes. The metric is in fact been worked out in details in [13] and therefore we shall be brief. The ranks of the fluxes can actually be incorporated in the background metric by choosing different harmonic functions along various directions in M-theory. If we denote the harmonic functions by $H_{i}$, with $i=0,1,2,3$, then the metric of the Taub-NUT space modifies from the usual form to

$$
d s_{T N}^{2}=f^{\frac{1}{3}}\left(d r^{2}+r^{2} d \Omega^{2}\right)+H_{0} f^{-\frac{2}{3}}\left(d x^{7}+\frac{1}{2} R \cos \gamma d \phi+\ldots\right)^{2},
$$

where $(r, \gamma, \phi) \leftrightarrow x^{8,9,10}$ and the dotted terms denote the mixing between $d x^{7}$ and $d x^{0}$ as explained in [13]. We have also defined $f$ as $f=H_{1} H_{2} H_{3}$. All the harmonic functions $H_{i}$ are functions of $r, R$ and $\cos \alpha_{k}$ with $k=1,2,3$ and $R$ is related to the radius of the TN space. The angles $\alpha_{k}$ are related to the twists of the T-dual tori as shown in [13]. Now a single centered TN space supports a unique harmonic form $\omega$, which, in the presence of fluxes can be written in terms of the above set of coordinates as $d \zeta$, where

$$
\zeta=g(r)\left(d x^{7}+\frac{1}{2} R \cos \gamma d \phi\right)
$$

with $\omega$ satisfying a normalisability condition such that it is anti self-dual. For the most generic case when we have at most four different warp factors denoted by $H_{0,1,2,3}$ related to (as we shall see later on in this section) different ranks of the $B$ fields, we can write the function $g(r)$ as (in units of $2 \pi l_{p}^{2}$ )

$$
g(r)=\exp \left[-\frac{1}{2} R \int_{r}^{\infty} H_{0}^{\frac{1}{2}} H_{1}^{-\frac{1}{2}} H_{2}^{-\frac{1}{2}} H_{3}^{-\frac{1}{2}}\right] .
$$

It turns out that for this generic case a closed form estimate is rather difficult. However we can try the rank four case. The two form can now be computed exactly, the function $g(r)$ being given by (again in units of $2 \pi l_{p}^{2}$ ) :

$$
g(r)=\left(1+\frac{\cos \alpha_{2}}{\cos \alpha_{1}}\right)\left(1+\frac{\cos \alpha_{1}}{\cos \alpha_{2}}\right) \frac{2 r}{\left(\sqrt{R+\frac{2 r \cos \alpha_{1}}{\cos \alpha_{2}}}+\sqrt{R+\frac{2 r \cos \alpha_{2}}{\cos \alpha_{1}}}\right)^{2}},
$$

where $R$ and $r$ are defined above. There are two cases now:

(1) $\alpha_{1}=\alpha, \quad \alpha_{2}=0$. This is the case that has been studied earlier and is used to get non-commutative theory on D6 branes [13]. We will discuss this soon. 
(2) $\alpha_{1}=\alpha_{2}=\alpha$. In this case $g(r)$ is given by $g(r)=8 r(R+2 r)^{-1}$, and is independent of the angle $\alpha$. However this value of $g(r)$ only gives approximate results for the noncommutative case. This will be elaborated later.

In this section we will see how, many interesting dynamics of oriented $B$ fields can arise directly from the TN configuration with the simplest choice of $g(r)$. We begin with the pinned brane scenario where we will also have a M5 brane in the TN configuration.

\subsection{Pinned Branes}

The Taub-NUT space is along directions $\left(x^{7}, x^{8}, x^{9}, x^{10}\right)$, where $x^{7}$ is compact circle whose radius $R$ shrinks to zero at the origin $r=0$. An M5 brane is kept at $r=0$ orthogonal to the TN space and is oriented along $\left(x^{0}, x^{1}, \ldots, x^{5}\right)$. We identify $x^{1}$ to the Mtheory circle. Now we switch on a 3 -form background $C_{167}$ which is a constant at infinity and is a function of $r$ given by (in units of $M_{p}^{3}$ )

$$
C_{3}=\frac{C\left(1+C^{2}\right)}{C^{2}+\left(1+\frac{R}{r}\right)} d x^{1} \wedge d x^{6} \wedge\left(d x^{7}+A_{i} d x^{i}\right)
$$

where $\mathrm{C}$ is the expectation value of the 3 -form at infinity and $A_{i}$ are the Taub-NUT gauge fields. It turns out that if the M5 brane has a tension $T_{0}$ at infinity, then the tension at the origin of the TN is $T=T_{0}\left(1+C^{2}\right)^{-\frac{1}{2}}$. Therefore the brane is pinned at the origin.

This pinning of the brane gave mass to four scalars of the $(2,0)$ theory. The fermion mass puzzle was solved by observing that the theory has only a $(4+1)$ - dimensional poincare invariance and therefore only $(4+1)$-d supersymmetry. In $(4+1)$ - d a fermion can be given a mass.

Another apparent puzzle arises when we try to study the near horizon geometry of the M5 brane. According to [1], near $r=0$ we can scale the coordinate $x^{1} \rightarrow x^{1} \sqrt{1+C^{2}}$ such that Lorentz invariance is seemingly restored. One might expect that the near horizon geometry will be $A d S_{7}$. This would also imply that the theory is scale invariant and the mass of the hyper is zero. But a calculation for fermions show that they are not massless. The resolution is that the scaling of coordinates which generated Lorentz invariance makes no sense for a point arbitrarily close to $r=0$. A small fluctuation of the M5 brane will break Lorentz invariance in this picture and also conformal invariance generating a scale $m$ such that

$$
m=\frac{C}{\sqrt{1+C^{2}}} .
$$

Therefore, for any finite $C$ the theory no longer has any $A d S$ limit. 
When the external parameters were carefully chosen this lead to new theories in six dimensions which were decoupled from gravity. The first decoupled theory can be seen when the external parameters are:

$$
C \rightarrow \epsilon, \quad R_{7} \rightarrow \epsilon, \quad M_{p} \rightarrow \epsilon^{-\beta}, \quad \beta>1
$$

In this limit the energy scale of the excitations of the M5 brane is kept finite whereas the other scales in the problem are set to infinity. This decoupling is kinematical. For a different scaling of external parameters

$$
C \rightarrow \text { finite }, \quad R_{7} \rightarrow \text { finite }, \quad M_{p} \rightarrow \infty, \quad g_{s} \rightarrow 0
$$

we get a dynamical decoupling. This decoupling is in the same spirit as the little string theory.

\subsection{Dipole Theories}

To get dipole theory from the configuration studied above is easy. Instead of orienting the M5 along $x^{0,1,2,3,4,5}$, we now orient the M5 along $x^{0,1,2,3,4,6}$ such that the background $C$-field $C_{167}$ will have two of its components along the M5 brane. In type IIA side we have a D4 brane along $x^{0,2,3,4,6}$ with a $B$ field $B_{16}$ along the brane. This case have been studied in much detail in [2,5,4,7] and therefore we shall be brief and only mention some of the salient features. A D4 brane oriented along $x^{0}, x^{2}, \ldots, x^{5}$ and orthogonal to a Taub-NUT space along $x^{7}, \ldots, x^{10}$ is pinned by a pinning potential given by

$$
\frac{\sqrt{\operatorname{det} g}}{g_{s}}=\cos \theta=\frac{1}{\sqrt{1+b^{2}}}
$$

in the presence of a $B_{N S}$ field, with an asymptotic value of $b$, and oriented along $x^{6}, x^{7}$ directions as

$$
B_{N S}=h \sqrt{f}_{2} \tan \theta d x^{6} \wedge\left(d x^{7}+B_{7 i} d x^{i}\right)
$$

where the string coupling $g_{s}=e^{\phi}=\sqrt{h f_{1} f_{2}^{-1}}$ and $f_{1,2}$ are the harmonic functions for this background with metric components denoted by $g_{i j}$ and string coupling $g_{s}$. The $B_{N S}$ field asymptotes to $\tan \theta \equiv b$. In the above calculations we have defined a quantity $h$ as

$$
f_{2}^{1 / 2} h^{-1}=f_{2} \sin ^{2} \theta+f_{1} \cos ^{2} \theta
$$

which determines how the Taub-NUT circle behaves in the presence of the $B_{N S}$ field. As its known, the Taub-NUT circle is non trivially fibered over the base and the metric of the 
fibration involves $h$ linearly. When we make a T-duality along $x^{4}$ the dilaton changes to

$\phi \rightarrow \phi-\frac{1}{2} \log g_{44}$ and therefore a D3 brane is also pinned when $B_{N S}$ field is orthogonal to it. The pinning potential is the same.

This part of the story is well known and therefore we should ask what happens for the other case, i.e. when we have a D4 oriented along $x^{0,2,3,4,6}$ at the $r=0$ point of TN space along $x^{7,8,9,10}$ and a $B_{67}$ field on it. As shown in [5] there is no pinning potential now, but the theory is governed by a new star product called the dipole star product. Many new aspects of this theory have been studied in [14] using supergravity analysis and in [15] from the pp-wave analysis.

An alternative way to get dipole theories on branes is to use duality twist discussed in [4. Equivalence between this and the one got from the Taub-NUT example is shown in [7]. Furthermore there are two interesting aspects of these theories not so intuitive:

(a) There exist a scale - the dipole length - in these theories. Yet the Beta function is zero. This is also reminiscent for the case of noncommutative geometry.

(b) The theory is non-renormalisable in the usual sense because there are in principle, infinte counterterms. However the form of the counterterms are exactly determinable at all loop orders.

\subsection{Noncommutative Geometry}

However in both the cases above we have kept the value of $C$ very low. An interesting case is when $C \rightarrow \infty$ and we remove the M5 brane from the picture. We also identify the M-theory cycle as $x^{7}$ instead of $x^{1}$. In type IIA we will therefore have a D6 brane oriented along $x^{0,1,2,3,4,5,6}$ with a $B$ field.

Let us first consider the rank two case. We have the $B$ field oriented along $x_{1,6}$ which we denote by $B_{16}$. When we lift this to M-theory we have a threeform $C_{167} \equiv C$ where $x^{7}$ is the 11th direction as mentioned above. This threeform will backreact on the geometry and will change the value of $g(r)$ as calculated for the generic case in (eq. 2.4) . For our case the value of $g(r)$ have been worked out earlier in [13] and can also be derived directly from (eq. 2.4) assuming $\alpha_{1}=\alpha, \alpha_{2}=0$. It is given by

$$
g(r)=\frac{2 r(1+\cos \alpha)(1+\sec \alpha)}{(\sqrt{R+2 r \cos \alpha}+\sqrt{R+2 r \sec \alpha})^{2}} .
$$

Using this value of $g(r)$ and considering the following limits of the background fields (see also [16]):

$$
C \rightarrow \infty, \quad M_{p} \rightarrow \infty, \quad M_{p}^{3} C^{-1} \rightarrow \text { fixed }
$$


we get a $6+1$ dimensional noncommutative YM theory whose coupling constant

$$
g_{Y M}^{2}=M_{p}^{-3} C=\text { fixed }
$$

This limit is consistent with (and in fact it's the same as) the limit studied by SeibergWitten [10]. A way to see this would be as follows:

The SW limit for a D6 brane with a rank 2 dimensionless $B_{\mu \nu}$ field is given in terms of dimensionful type IIA metric $g_{\mu \nu}$. If $l_{s}$ is the string length and $g_{s}$ is the string coupling constant the limit is

$$
l_{s} \rightarrow \epsilon^{1 / 4}, \quad g_{\mu \nu} \rightarrow \epsilon, \quad g_{s} \rightarrow \epsilon^{-1 / 4}
$$

where $\epsilon \rightarrow 0$ is used to parametrise very small quantities. We now want to study this limit from M-theory point of view. Recall that we have identified the eleven dimensional circle with the Taub-NUT circle, i.e with $y$ and taken the length dimensions in M-theory as

$$
\left[g_{m n}^{M}\right]=0, \quad\left[C_{m n p}\right]=0, \quad[\partial]=-1
$$

where $g_{m n}^{M}$ is the $11 \mathrm{~d}$ metric. Invoking now the usual relations between the IIA variables and M-theory variables, we are led to the following limit:

$$
R_{y} \simeq g_{s}^{2 / 3} l_{p} \simeq g_{s} l_{s}=\text { constant }, \quad l_{p} \rightarrow \epsilon^{1 / 6}
$$

where $R_{y}$ is the radius of the Taub-NUT circle. This would imply that in our limit when $l_{p}^{3} C$ is a constant,

$$
C \rightarrow \epsilon^{-1 / 2}
$$

and the type IIA variables $B_{\mu \nu}$ and $g_{\mu \nu}$ are related to the dimensionless $C$ field as $C=\frac{\alpha^{\prime} B}{g}$. A consistency check of this would be to note that

$$
g_{Y M}^{2}=g_{s} l_{s}^{3} \sqrt{\operatorname{det}\left(\frac{\alpha^{\prime} B}{g}\right)} \rightarrow \text { constant }
$$

agrees with the identification. Therefore to summarise, we get a $6+1$ dimensional noncommutative YM for the following limit of the external parameters:

$$
C \rightarrow \epsilon^{-1 / 2}, \quad M_{p} \rightarrow \epsilon^{-1 / 6}, \quad R_{y} \rightarrow \text { constant }
$$

Observe that the $C$ field in other directions are not excited. The dimensionless M-theory metric, $g_{m n}^{M}$, now scales differently. Along the directions of non-commutativity they scale as

$$
g_{55}^{M} \rightarrow \epsilon^{2 / 3}, \quad g_{66}^{M} \rightarrow \epsilon^{2 / 3} .
$$


Along other directions they scale in the following way:

$$
g_{\mu \nu}^{M} \rightarrow \epsilon^{1 / 6}, \quad g_{y y}^{M} \rightarrow \epsilon^{-1 / 3} .
$$

It is easy to check that $R_{y}^{2}=l_{p}^{2} g_{y y}^{M} \rightarrow$ constant as expected.

\section{The rank four and rank six cases}

To study the rank $r$ case, we will use the fact that string coupling scales as

$$
g_{s} \rightarrow \epsilon^{\frac{r-3}{4}}, \quad \text { and } \quad l_{s} \rightarrow \epsilon^{1 / 4} .
$$

An obvious invariant that could be made from above scaling is the combination: $g_{s} l_{s}^{3-r}$. Indeed this is exactly what is kept fixed in the non-commutative theory and is related to the $g_{Y M}^{2}$. In the M-theory description we have

$$
R_{y} \rightarrow \epsilon^{\frac{r-2}{4}}, \quad \text { and } \quad l_{p} \rightarrow \epsilon^{r / 12} .
$$

This immediately implies that for the higher rank fields we really do not need the 11dimensional picture at the decoupling limit. This is also consistent with earlier results [17]. We notice also that the 11-dimensional Planck length does go to zero for rank 4 and 6 cases too. The YM coupling constant is given in terms of M-theory variables as: $l_{p}^{3} C^{r / 2}$. This implies

$$
C_{i} \rightarrow \epsilon^{-1 / 2}, \quad \text { and } \quad \sqrt{\operatorname{det}\left(\frac{\alpha^{\prime} B}{g}\right)} \rightarrow \epsilon^{-r / 4},
$$

where $i=2, . ., r$ and therefore $g_{Y M}^{2}$ is a constant. The metric along the non-commutativity directions now scale as

$$
g_{i j}^{M} \rightarrow \epsilon^{\frac{6-r}{6}}
$$

and the dimensionless and the dimensionful metrics in the 11th direction $y$ scale as

$$
g_{y y}^{M} \rightarrow \epsilon^{\frac{r-3}{3}}, \quad g_{y y}=l_{p}^{2} g_{y y}^{M} \equiv R_{y}^{2} \rightarrow \epsilon^{\frac{r-2}{4}} .
$$

Along other directions they scale as

$$
g_{m n}^{M} \rightarrow \epsilon^{\frac{3-r}{6}},
$$

implying that the metric scales differently along different directions of the brane as expected. Similar behaviour can be seen for the dipole theory also which was mentioned in [5]. 
2. $\underline{l_{p} \rightarrow 0 \text { limit }}$

In the M-theory description we define various scales as

$$
2 \kappa_{11}^{2}=(2 \pi)^{5} l_{p}^{9}, \quad \kappa_{10}=\frac{8 \pi^{4} l_{p}^{9}}{R_{y}}, \quad T=\frac{1}{2 \pi l_{p}^{3}},
$$

where $T$ is the membrane tension. The coefficient of the bosonic part of the $11 \mathrm{~d}$ supergravity is $-\frac{1}{2 \kappa_{11}^{2}}$ if we take the following length dimensions

$$
[g]=0, \quad\left[C_{i}\right]=0, \quad[\partial]=-1 .
$$

To see whether the gauge kinetic term survives let us consider the kinetic term of the three-form field. It is given by

$$
\frac{1}{2 \kappa_{11}^{2}} \int G \wedge *{ }_{11} G \rightarrow l_{p}^{-9} \int \sqrt{g} G_{\mu \nu \rho \sigma} G_{\mu^{\prime} \nu^{\prime} \rho^{\prime} \sigma^{\prime}} g^{\mu \mu^{\prime}} g^{\nu \nu^{\prime}} g^{\rho \rho^{\prime}} g^{\sigma \sigma^{\prime}}
$$

where $g_{\mu \nu} \equiv g_{\mu \nu}^{M}$ is the dimensionless M-theory metric. If we require the gauge fields to be along directions $x_{1,2,3,4}$ then a typical term will look like

$$
l_{p}^{-9} \sqrt{g} g^{11} g^{22} g^{m m^{\prime}} g^{n n^{\prime}} G_{12 m n} G_{12 m^{\prime} n^{\prime}} .
$$

It is easy to see that this term scales as

$$
\epsilon^{-1 / 2} g_{s}^{-1} l_{s}^{-3},
$$

which is a constant implying that the gauge kinetic term survives the scaling.

Consider now the next higher order term

$$
T \int \sqrt{g} R^{h m n k} R_{p m n q} R_{n}{ }^{r s p} R_{r s k}^{q} \sim l_{p}^{-3} \sqrt{g}\left(g^{-1}\right)^{8} R^{4} .
$$

Each $R$ scales as either $\epsilon^{2 / 3}, \epsilon^{1 / 6}$ or $\epsilon^{-1 / 3}$ depending on the orientation. In fact it is easy to see that

$$
\epsilon^{1 / 6} \leq\left(g^{-1}\right)^{8} \leq \epsilon^{-23 / 6}, \quad \epsilon^{8 / 3} \leq(R)^{4} \leq \epsilon^{-4 / 3} .
$$

This term decouples in the path integral and therefore do not contribute to the quantum fluctuations. It would be interesting to study the behaviour of other higher order terms. However since the explicit forms of these terms are not known, it is difficult to see what happens under the scalings.

For this theory we could calculate various BPS states. The masses of these states are all proportional to $\left(M_{p}^{3} / C\right)^{\alpha}$. We get light M2 branes for $\alpha=1$ and light M5 branes for $\alpha=2$. 


\section{Theory Near Conifold Type Geometry}

In the previous section we studied an M5 brane near a Taub-NUT singularity. Let us reorient the system such that the M5 is oriented along, say, $x^{0,1,2,3,4,9}$ and the TN along $x^{6,7,8,10}$ with $x^{7}$ being the TN circle and the other three directions non-compact. Furthermore the directions $x^{5,9}$ are also non-compact. Assuming now that the directions $x^{5,9}$ have a topology of a sphere $P^{1}$ the ALE space will have the following form:

$$
z_{1}^{2}+z_{2}^{2}+z_{3}^{2}=-|\mu|^{2}
$$

where $z_{1,2,3}$ are used to denote the TN space and $\mu$ is the size of the blown up sphere $P^{1}$. If we now identify $\mu=z_{4}=x^{5}+i x^{9}$ then this is the equation of a conifold oriented along $x^{5,6,7,8,9,10}$. Thus we get a way to study branes near conifold singularities using the approach discussed in the previous section!

To be a little more precise, we actually need a configuration of M5 brane near a conifold type singularity in the presence of fluxes. To achieve the supergravity description of this we take a system of intersecting D5 and NS5 branes in type IIB theory. The D5 brane is oriented along $\left(x^{0}, x^{2}, \ldots, x^{4}, x^{7}, x^{9}\right)$ and we have two NS5 branes oriented along $\left(x^{0}, x^{2}, \ldots, x^{6}\right)$ and $\left(x^{0}, \ldots, x^{4}, x^{8}, x^{9}\right)$ respectively. We will further assume that directions $x^{6}, x^{7}$ are on a slanted torus whose angle is $\theta$. The direction $x^{1}$ will be the M-theory direction. By T-dualising along $x^{7}$ we get a configuration in type IIA theory 1 , which when lifted to M-theory along $x^{1}$ will reproduce the background that we are interested in.

\subsection{Pinned Branes}

The interesting thing now is that the harmonic functions are all linear functions of the overall transverse direction, which is $x^{10}$ in our case. This is however assuming that the D5 brane is completely delocalised along the NS5 branes' directions.

We have oriented the $\mathrm{D} 5$ brane in such a way that the theory on it will be a $U(1) \times U(1)$ gauge theory. The product gauge group arises simply because the D5 brane is "cut" twice by the two NS5 branes. The theory will now have a lower supersymmetry. The metric for

1 A slight variant of this problem was studied earlier in 18, 19 where a conifold in type IIB theory was shown to be T-dual to two intersecting NS5 branes. Another related construction was given in [20] where a conifold in type IIB theory was shown to be T-dual to two intersecting NS5 in type IIB theory giving rise to the so-called brane boxes [21]. These construction (in the IIA picture) were used, in a different context, to understand the geometric transition in $\mathcal{N}=1$ theories [22]. Although unrelated to the main line of thought presented here, the Taub-NUT background also played a very crucial role there. We will however not discuss this anymore in the paper. 
this configuration will be same as of D5-NS5-NS5' branes but with a slight deformation along the $x^{6}, x^{7}$ directions because of the slanted torus. There will be a cross term in the metric which as seen by the D5 brane will be

$$
2\left(1+\left|x^{10}\right|\right) \tan \theta d x^{6} d x^{7} .
$$

The reason we choose this configuration is because by making a T-duality along direction $x^{7}$ we will get a configuration of a $\mathrm{D} 4$ brane at a conifold point in type IIA. The conifold in question arises from the two intersecting NS5 branes which overlap along three common directions. We will also get a NSNS $B$ field background fom the slant of the torus. Note that the two NS5 branes also contribute to NS $B$ fields. We can gauge away most of the components of the source $B$ fields and keep only $B_{78}$ and $B_{75}$ from the two NS5 branes respectively. Therefore the background now looks like

$$
B=\frac{\tan \theta}{\sin ^{2} \theta+\left(1+\left|x^{10}\right|\right)^{q} \cos ^{2} \theta} d x^{6} \wedge\left(d x^{7}+B_{78} d x^{8}+B_{75} d x^{5}\right),
$$

where $q=1$ when we have the $\mathrm{D} 4$ as a probe, and $q=0$ when the effect of $\mathrm{D} 4$ is completely delocalised.

It is important to note that we actually get a conifold for values of $\left|x^{10}\right|$ sufficiently small. For this limit all the harmonic functions are essentially constant and the metric looks conformal to a conifold under some scaling. Another crucial thing which is necessary to reproduce the conifold geometry is that directions $x^{4,5}$ and $x^{8,9}$ are spheres. However for our case we will not take these directions as spheres, instead they will be toroidal. Therefore the geometry is conifold like (meaning that it falls in the same equivalent class as conifold geometry).

We now lift this configuration to M-theory. The parameter which lifts the metric is the type IIA coupling given by

$$
e^{2 \phi}=\frac{\left(1+\left|x^{10}\right|\right)^{p}}{A \sin ^{2} \theta+B\left(1+\left|x^{10}\right|\right)^{q} \cos ^{2} \theta} .
$$

There are three interesting cases now:

(1) In the absence of probe branes, $A=B=p=q=1$. This is the usual case that we shall be concentrating mostly. This behaviour persists when we take the effect of probe on the background to be very small.

(2) When we assume full delocalisation in the presence of probe branes $A=B=1, q=$ $0, p=-\frac{3}{2}$. This case is also similar to the case when we have $q=0$ in (eq. 3.3). The 
delocalisation effect dilutes the background considerably and therefore most of the effects are actually washed out, for example the non-trivial behaviour of $B$-field.

(3) Assuming partial delocalisation, $p=q=1$ and $A=f^{\frac{5}{2}}, B=f^{\frac{3}{2}}$; where $f$ is the localised harmonic function for the probe. This is a more involved case and we will have nothing new to say here.

In the absence of any background B-field $e^{2 \phi}=1$ and therefore the M-theory and the type IIA metric is the same. For this case we see that we can come back to type IIA via the $x^{7}$ circle (instead of $x^{1}$ ). But now we will get a configuration of two intersecting D6 branes (see also [23]). This information can also be used to verify the results.

Another important thing to note is that the M5 brane (which is the lift of the D4 brane) will only see a $3+1$ dimensional poincare invariance. This is because of our choice of the two intersecting NS5 branes. The M5 brane overlaps with four of the "flat" directions of the conifold but one of the directions $\left(x^{1}\right)$ is scaled differently.

If $T_{0}$ is the tension of the M5 brane in the absence of the background $B$ field then near the origin $\left|x^{10}\right| \rightarrow 0$ we have a M5 brane with tension $T_{0}$ and near $\left|x^{10}\right| \rightarrow \infty$ we have a $\mathrm{M} 5$ brane with tension $T_{0} \cos \theta$.

Let us consider a case in which the slant of the torus $\theta \rightarrow \pi / 2$. In this situation, writing the metric components as $g_{i j}$ before, we observe that for all finite values of $\left|x^{10}\right|$

$$
\text { det } g=g_{00} g_{11} g_{22} g_{33} g_{44} g_{99}=\sin ^{2} \theta+\left(1+\left|x^{10}\right|\right) \cos ^{2} \theta \rightarrow 1
$$

This result seems to suggest that a M5 brane will now see a completely flat potential! Therefore any pinning in the absence of $C$ is completely removed in this limit. However the way we have motivated the model - starting with D5 -NS5 -NS5' system - the D5 is stuck at the zero of the coulomb branch by the construction itself. There is of course the Higgs branch but that motion will give rise to some massive states in the gauge theory. Observe that for the case of M5 brane near a Taub-NUT space, even for $C \rightarrow \infty$, this effect will never be there. In fact we will get the maximum pinning for this limit. The tension of the M5 brane will remain 1 at $r \rightarrow \infty$.

Thus we seem to be getting the following salient features from our model:

(1) Theory on the M5 brane (or D4 brane from IIA point) has only $3+1$ dimensional poincare invariance. The $4+1 \mathrm{~d}$ poincare invariance here is broken by the geometry of the construction, as against the model studied earlier, where the poincare invariance was broken by the background $C$ field.

(2) From construction we have a M5 brane "pinned" at the origin. But now, for the choice of large background or $\theta \rightarrow \pi / 2$, we can keep another M5 brane anywhere in the spacetime, 
which would not be possible for $\theta=0$. This effect is completely opposite of the effect we saw in the previous case. Here we are getting an unpinning of the brane because of the presence of large $C$ field. This may be used to study models analogous to the the Braneworld scenario where the starting point is by keeping a mirror brane at a distance $r_{c}$ from the original brane and then take the limit of $r_{c} \rightarrow \infty$.

(3) The theory on the M5 brane now has only $N=1$ supersymmetry in the poincare invariant $3+1$ dimensional space.

One can continue along these lines to study more interesting aspects of pinned (or "unpinned") branes. As such it is not clear whether the pinning effect remains when we have a configuration which breaks supersymmetry completely. For the conifold like singularity we do not have an identical BPS calculation, which was done for the TaubNUT case, to support our observation of unpinning. It will be interesting to find an alternate confirmation of this.

\subsection{Hybrid Theory: Noncommutative Geometry and Dipole Theory}

In the above analysis we briefly mentioned that we could get intersecting six-branes in type IIA theory if we reduce the conifold geometry along some other direction. Let us recapitulate the issue. We started with a configuration of a D3 brane near two intersecting NS5 branes. The metric of the system is more or less identical to the metric of a single D3 brane without any other effects. The back-reaction of the NS5 branes can be incorporated by shifting the coordinates $d x^{i}$ to

$$
d x^{i} \rightarrow\left(1+\left|x^{10}\right|\right)^{p / 2} d x^{i}
$$

where $p$ can be either 0,1 or 2 depending on the directions. As discussed earlier, the background also have $B$ fields that are sources of the NS5 branes. For simplicity if we assume that these backgrounds are unity for some appropriate choice of scales, then it is easy to show that the conifold cycle in M-theory (which we denote by $d \psi$ ) becomes

$$
d \psi=\frac{d x^{7}+\cos \theta_{1} d x^{8}+\cos \theta_{2} d x^{5}}{\left(1+\left|x^{10}\right|\right)^{\frac{2}{3}}\left(1+\left|x^{10}\right| \cos ^{2} \theta\right)^{\frac{1}{3}}},
$$

where $\tan \theta$ measures the value of the antisymmetric field at infinity and $\theta_{1,2}$ measure the values of B-fields $B_{78}$ and $B_{75}$. The above way of writing also guarantees that the M-theory background can be put in a simple form as

$$
d s^{2}=d \psi^{2}+e^{4 \phi / 3}\left(d x^{1}\right)^{2}+e^{-2 \phi / 3}\left[-\left(d x^{0}\right)^{2}+\left(d x^{2}\right)^{2}+\ldots\right],
$$


where we have used the scalings given in (eq. 3.6). There is a small subtlety though. In the presence of G-fluxes, the direction $d x^{6}$ is further suppressed by sec $\theta$, even though rest of the components follow the rule given in (eq. 3.6) .

At this point we have two possibilities to come down to type IIA theory: either along $x^{1}$ or along $x^{7}$. The first possibility is of course the one dealt in the previous section, wherein we get conifold singularity in the type IIA side. The novel thing is the other way of doing it. It is well known that in the absence of any fluxes, reducing M-theory on $x^{7}$, will give us two intersecting D6 branes [23]. What happens in the presence of fluxes? We will indeed get two intersecting D6 branes, but now due to the presence of fluxes, one of the branes will have non-commutaive star product whereas the other one will have dipole star product! This is what we call as Hybrid Theory. Question is how is this consistent, when, on one side spacetime coordinates do-not commute whereas the other side they do?

To answer this, observe that we get two D6 branes oriented along $x^{0,1,2,3,4,5,6}$ and $x^{0,1,2,3,4,8,9}$ with a curved metric that can be easily determined from the above analysis?. There would be the KK gauge fields that would come directly from the cross terms discussed in the metric component $d \psi$. These KK gauge fields are of course the D6 brane sources. There would also be RR three forms $C_{168}$ and $C_{165}$ plus an antisymmetric two form

$$
B_{16}=\frac{\tan \theta}{1+\left|x^{10}\right| \cos ^{2} \theta}
$$

This is the two-form that is responsible for generating a hybrid theory. Observe that now, even though we do not have any TN background, this $B$ field cannot be gauged away because it lies on both the branes. Because of the orientation of the two D6 branes there is no conflict due to spatial non-commutativity as the directions of non-commutativity donot overlap completely. In other words, the direction $x^{1}$ and $x^{6}$ are non-commutative but $x^{6}$ do not lie on the other brane. Since $x^{1}$ commutes with $x^{2,3,4,8,9}$ directions on the other D6 brane, it doesn't violate the fact that this theory can now have dipole star product.

2 For the case when we have two NS5 branes oriented along $x^{0,2,3,4,5,6}$ and $x^{0,2,3,4,8,9}$, an Sduality transformation will give us two intersecting D5. Now T-dualising along $x^{7}$ we will get two intersecting D6 branes in type IIA theory. Lifting this to M-theory along the M-theory direction $x^{1}$, this will give a conifold like geometry. The relation between the intersecting D6 that we have here and the one got by S-duality of the NS5 is simply the interchange of $x^{1}, x^{7}$ direction in M-theory. As is well known, this is how the S-duality shows up in M-theory. 


\section{Theory Near Multi-Centered Taub-NUT Geometry}

Till now we have either taken a single centered Taub-NUT space or a Taub-NUT space fibered over a $P^{1}$ base forming a conifold. It is time now to go to multi-centered TN space in the presence of fluxes and see whether we can extend the previous analysis to this level also. We could take, as before, an M5 brane near such singularities (in the presence of oriented fluxes) or remove the M5 brane and study the configuration directly in type IIA picture. Let us first consider the case when we have no M5 branes. Let us also assume that all the TN singularites are at the point $r=0$.

In type IIA theory this therefore gives rise to multi D6 branes stacked at the point $r=0$. In the presence of large number of $\mathrm{D} 6$ branes there is an inherent non-commutativity of the space-time coordinates because of the non-abelian nature. This is reflected from the fact that matrices don't commute. Let us now switch on a $B$ field to generate further noncommutativity. However these two noncommutativity do not mix in any usual sense and the product rule is specified by a simple tensoring of constant matrix algebra and the Moyal-Weyl deformation

$$
(\Phi * \Psi)_{k}^{i}(X)=\sum_{j}\left(\Phi_{j}^{i} * \Psi_{k}^{j}\right)(X)
$$

This is however not quite the case when we have multiple D-branes each seeing a different B-field on its world volume[6]. A way to configure such a system in string theory is to have multiple D-branes in a spatially varying B-field 3 . The pull back of the B-field on each brane is constant. In the setup that we described earlier, this could be realised by switching on a $G$-flux in the multi Taub-NUT background. The $G$-flux has non-zero expectation values only near the Taub-NUT singularities [6]. This configuration may or may not preserve any supersymmetry depending on the configuration. Later on we shall give a concrete example where an $\mathcal{N}=1$ susy is preserved.

As it turns out, a two point lattice approximation to open string is perfectly suited for this 12]. We can get away with the enormously complex nature of products of string wave functions while retaining the essential nature of the non-commutativity of such products. Therefore, though the calculations are motivated from string theory, the lattice string quantum mechanics (LSQM) is an independent way to calculate this product. More clearly, what we need is the following decomposition rule for a string wavefunction $\Psi(x)$ to the dipole basis $e_{1,2}$

$$
\Psi\left(e_{1}^{a}, e_{2}^{a}\right)=\int d X^{\prime} \Psi\left(X^{\prime}\right)\left\langle X^{\prime} \mid e_{1}^{a}, e_{2}^{a}\right\rangle,
$$

3 Another way would be to switch on different gauge fields $F_{i}$ on the $i^{\text {th }}$ brane. In the presence of both $F$ and $B$ the invariant quantity is $\mathcal{F}_{i}=F_{i}-B_{i}$. Henceforth we will just specify $\mathcal{F}_{i}$. 
where $\left\langle X^{\prime} \mid e_{1}^{a}, e_{2}^{a}\right\rangle$ is the change of basis function that we shall discuss in the next few sections. A way to evaluate this is given in [6,12]. Using this one can show that the new product is no longer a simple tensoring of the star product (eq. 4.1) and non-abelian matrix algebra. The noncommutative real space and the non-Abelian internal space get intertwined and inseparable. This is the main idea of nonabelian geometry [6]. The deformation equation becomes:

$$
(\Phi \times \Psi)_{k}^{i}(X) \equiv \sum_{j}\left(\Phi_{j}^{i} *_{i j k} \Psi_{k}^{j}\right)(X)
$$

where $*_{i j k}$ depends on all the three indices. Let us now discuss the various possibilities.

\subsection{Pinned Branes And Dipole Theory}

What we now require is that a M5 brane should probe this background. In IIA theory this is nothing but a D4 probing a multi TN background, in the presence of $B$ flux that could either be completely orthogonal to the brane or should have one leg along the brane. For the case when we have the $B$ field completely orthogonal to the brane, it is determined in terms of $\alpha$ as

$$
B=\frac{1+a \cos ^{2} \alpha}{1+b \cos ^{2} \alpha} B_{o},
$$

where $B_{o}$ is the $B$ field for the single centered TN. The factors $a, b$ are in general functions of the $r$ and the points where the TN circles would degenerate, the explicit form of which can be easily determined from [1]. Question now is what would happen to the probe M5 brane as it goes towards any degenerating points of the multi TN space. One can argue that at any such points the backreaction due to the harmonic functions is very large and therefore the M5 brane is effectively pinned at that point. Therefore the multi TN space has many fixed points for the M5 brane and once the brane is fixed at one such fixed point it will not move to the other fixed point. Similar analysis can be performed for the dipole theory. One can show that the branes are not pinned at any points on the multi TN space and therefore the dipole behavior is not quite different from the case of single centered TN space.

\subsection{Noncommutative Geometry}

To understand the noncommutative theory from the point of view of M-theory, we can use the earlier configuration but now replace the background with a multi-TN space, as 
discussed above. In a $N$-centered TN space there are accordingly $N$ different normalisable harmonic forms $\omega_{i} \equiv d \zeta_{i}$ satisfying

$$
\int_{T N} \omega_{i} \wedge \omega_{j}=(16 \pi m)^{2} \delta_{i j},
$$

where $m$ measures the periodicity $16 \pi m$, of the TN circle so that there is no conical singularities at any point where a circle would degenerate. The generic description in the presence of fluxes would basically follow the arguments developed earlier and in [13]. For the rank four case (calling the harmonic functions for the background as $H_{0,1,2}$ ), the gauge coupling constant would be different for the different directions of the $B$ fields. For gauge fluctuations completely orthogonal to the noncommutativity direction, an estimate for the coupling is given by the following integral over the TN space:

$$
\int_{T N} H_{0} H_{1}^{-\frac{1}{2}} H_{2}^{-\frac{1}{2}} \omega \wedge * \omega,
$$

where the Hodge star is over the four dimensions. This integral is approximate because of the reasons mentioned in [13] but give a rough estimate of the result. For the rank two case this integral does indeed reproduce the correct coupling as mentioned in [13]. Now for the gauge fluctuation along the noncommutativity direction, an estimate for the coupling constant can now be done by the following integral over the TN background:

$$
\int_{T N} H_{0}^{-1} H_{1}^{\frac{3}{2}} H_{2}^{-\frac{1}{2}} \omega \wedge * \omega
$$

with a similar estimate for the other directions. This estimate improves when we consider rank two case. When we consider the case where all the ranks of the $B$ fields are the same, (eq. 4.7) will predict a coupling $l_{p}^{3} C^{2}$ for $g_{Y M}^{2}$, where $C$ is the expectation value of the bacground $C_{167}$ field. This is more or less what one would have expected for such a case, suggesting that the above integrals, though approximate, are not without merit.

Question now would be how to understand the open string behaviour directly from the TN geometry. This is easy if we assume that the points where we have shrunk two cycles, there are also wrapped M2 branes. These M2 branes will in fact appear on the D6 branes as open strings connecting two such D6 branes. We can take our open membrane with a cylindrical topology and with a coupling to a generalised three form $C+d B$. The boundary condition can be written down easily. Now we shrink the torus at two ends so that the topology is of a sphere as shown below: 


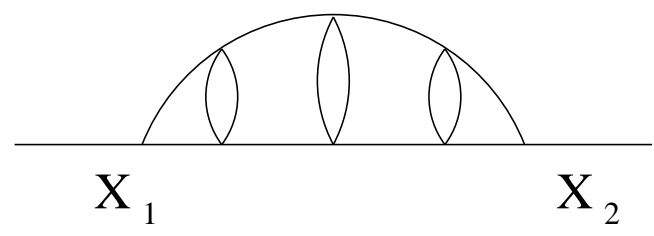

The boundary condition therefore simply becomes the boundary condition for open strings coupled to some gauge field. This situation is now ripe for the LSQM approach. The LSQM is the approximation wherein an open string is represented by two points, namely the two ends, and labelled by $X_{1}$ and $X_{2}$. The open string action now becomes the motion of a constrained dipole [12] $\left(2 \pi \alpha^{\prime}\right)^{2} G^{-1}$ remain finite and $G$ being the closed string metric, the result of canonical quantization with constraints is [6,24]

$$
\begin{aligned}
& {\left[X_{1}^{\mu}, X_{1}^{\nu}\right]=\imath \Omega_{i}^{\mu \nu},} \\
& {\left[X_{2}^{\mu}, X_{2}^{\nu}\right]=-\imath \Omega_{j}^{\mu \nu},} \\
& {\left[X_{1}^{\mu}, X_{2}^{\nu}\right]=0 .}
\end{aligned}
$$

From M-theory point of view these commutation relations are the one for the end points of an open membrane. Since the end points are shrunk to points, the commutation relations are simpler then their cousins. Using congruence transformation we can always transform $\Omega$ (or $\mathcal{F}$ ) into the following canonical form:

$$
\Omega_{i}=\left(\mathcal{F}^{i}\right)^{-1}=T_{i} J T_{i}^{\top} .
$$

The above way of writing will simplify the formulation of $*$-product in the subsequent analysis. Also notice from (eq. 4.8) $X_{1}$ and $X_{2}$ commutes among themselves. Actually in $R^{D}$ there are only $D / 2$ commuting coordinates at the two ends. Let us denote them as $e_{1}^{a}$ and $e_{2}^{a}, a=1,2, \ldots, D / 2$, where the subscript indicates boundary components. If $A, B, \ldots$ denote the rest of the coordinates then the canonical form $J$ is

$$
\begin{aligned}
J^{a A} & =\delta^{a+\frac{D}{2}, A}=-J^{A a} \\
J^{a b} & =0=J^{A B} .
\end{aligned}
$$

4 This dipole nature of the open strings should not be confused with the dipole theory discussed earlier. The dipoles of the dipole theory are the behavior of solitonic strings that are constrained to move in the background of $H_{N S}$ field. As a result of this motion, these strings tend to arch out of the brane on which the two ends are stuck. At a particular distances between the two ends of the solitonic strings the system is stable. These two ends form the dipoles of the dipole theory [5]. The dipoles discussed in this section and next are an approximation where the full string is represented by its two end points. 
Therefore the Hilbert space for LSQM is the tensor product of the Hilbert spaces of the two decoupled ends of the dipole. In this formulation, for wave functions $\Psi_{a a}\left(e_{1}^{a}, e_{2}^{a}\right)$ and $\Phi_{a a}\left(e_{1}^{a}, e_{2}^{a}\right)$ in the aa representation, the defination of $*$-product is straightforward:

$$
(\Psi * \Phi)_{a a}\left(e_{1}^{a}, e_{2}^{a}\right)=\int d M^{a} \Psi_{a a}\left(e_{1}^{a}, M^{a}\right) \Phi_{a a}\left(M^{a}, e_{2}^{a}\right),
$$

where the equality is upto a normalisation. This way of formulating the $*$-product, other than its intuitive appeal, has its root in the string field theory. It basically tells us the merging of two oriented paths into one as in string field theory [25] or, here, as the merging of two ordered pairs of points. To see that (eq. 4.11) reduces to the known formulation of the deformed product let us consider the case when

$$
\Omega_{i}=\Omega_{j}=\Omega
$$

As discussed above, since all the branes see the same noncommutativity parameter $\Omega$, we can convert $\Omega$ to its canonical form $J$. In this limit, since all $T_{i}$ 's are identity matrices,

$$
e_{i}=T_{i}^{-1} X_{1}, \quad e_{j}=T_{j}^{-1} X_{2}
$$

are just $e_{1}, e_{2}$, satisfying the following commutation relations:

$$
\begin{aligned}
& {\left[e_{1}^{\mu}, e_{1}^{\nu}\right]=J^{\mu \nu}=-\left[e_{2}^{\mu}, e_{2}^{\nu}\right],} \\
& {\left[e_{1}^{\mu}, e_{2}^{\nu}\right]=0 .}
\end{aligned}
$$

Now to apply (eq. 4.11) we have to define wave functions in terms of commuting coordinates. This can be readily shown to be the center of mass of the dipole

$$
X_{c}=\frac{1}{2}\left(X_{1}+X_{2}\right)=\frac{1}{2}\left(e_{1}+e_{2}\right)
$$

We are still a step behind using (eq. 4.11). What we now need is the change of basis function. Recall that the number of commuting coordinates are denoted by $a=1,2, . ., D / 2$ whereas $A, B, .$. denote the rest of the coordinates. The change of basis is therefore [12]

$$
\left\langle X_{c} \mid e_{1}^{a}, e_{2}^{a}\right\rangle=\delta\left(X_{c}^{a}-\frac{1}{2}\left(e_{1}+e_{2}\right)^{a}\right) \exp \left(-\frac{\imath}{4} X_{c}^{A} J_{A a}\left(e_{2}-e_{1}\right)^{a}\right)
$$

With this its now straightforward to use (eq. 4.11) . In terms of more general form $\Omega$ (eq. 4.11) is explicitly given by

$$
(\Psi * \Phi)(X)=\left.\exp \left(\frac{\imath}{2} \Omega^{\mu \nu} \frac{\partial}{\partial X^{\prime \mu}} \frac{\partial}{\partial X^{\prime \prime \nu}}\right) \Psi\left(X^{\prime}\right) \Phi\left(X^{\prime \prime}\right)\right|^{X^{\prime \prime}=X^{\prime}=X}
$$


which is precisely the deformed product appearing in noncommutative geometry [26,8, 10]. The above analysis can also be presented in the following graphical way:

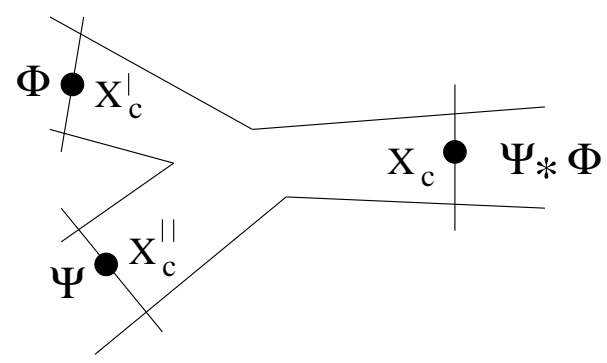

(A)

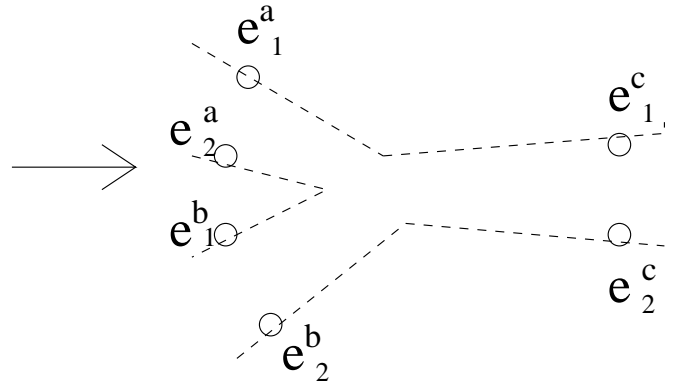

(B)

where figure (A) represents the way two wavefunctions $\Psi$ and $\Phi$ merge together to give the product wavefunction $\Psi * \Phi$ and figure (B) is the corresponding dipole way of viewing the above process. We have also shown the center of masses explicitly. The concept of center of mass will have important implications when we go to the next section.

\subsection{Nonabelian Geometry}

In the above analysis we didn't allow the nonabelian nature of the D6 branes to mix with the non-commutativity by choosing the same constant $B$ fields on all the branes. This may not be always the case. The nonabelian nature of the multiple D6 branes is apparent directly in the M-theory from the wrapped M2 branes. For this let us assume that the multi-TN space has many non-vanishing two cycles on which we can wrap M2 branes. Indeed the intersection numbers of all the wrapped M2 branes generate the following intersection matrix:

$$
\mathcal{I}=\left(\begin{array}{ccccccc}
2 & -1 & 0 & 0 & \ldots & 0 & 0 \\
-1 & 2 & -1 & 0 & \ldots & 0 & 0 \\
0 & -1 & 2 & -1 & \ldots & 0 & 0 \\
. & . & . & . & \ldots & . & . \\
. & . & . & . & \ldots & . & . \\
0 & 0 & 0 & 0 & \ldots & 2 & -1 \\
0 & 0 & 0 & 0 & \ldots & -1 & 2
\end{array}\right)
$$

as shown by Sen [27]. This intersection matrix is of course the Cartan matrix for $A_{N-1}$ singularity, which becomes $S U(N)$ gauge symmetry on $N$ D6 branes. Assuming now that we do not consider the case of coinciding D6 branes (we will eventually tackle the situation when we have coinciding system), the nonabelian geometry is realised now by the following choice of the background $G$ fluxes:

$$
\frac{G}{2 \pi}=\sum_{i=1}^{N} \mathcal{F}_{i} \wedge \omega_{i}
$$


where $\omega_{i}$ are the harmonic forms satisfying (eq. 4.5). In IIA point of view, this is then the setup where multiple D6 branes sees different amounts of noncommutativity on their world volumes. The reader, however, might still be concerned by the fact that at the coinciding stage the system might settle down to a configuration with a unique $\mathcal{F}$ field on its world volume. In the case that we study, this could in principle happen as a time dependent process but our main concern is not, for the time being, to study the final stage of this process. We will take this configuration to illustrate the possibility of a new star-product and then in the later part of the paper will give an example wherein such a configuration do occur. Therefore, in the language of D6 branes and LSQM we will assume:

$$
\Omega_{i} \neq \Omega_{j}
$$

as our starting point for nonabelian geometry. Now, as before, to use (eq. 4.11) we have to search for the commuting center of mass coordinate. Here comes the subtlety. The center of mass now is no longer the simple average of the two ends of the dipole, since such an average will not commute. However some small algebraic manipulations will give us the required $X_{c}$ as $[6]^{5}$

$$
X_{c} \equiv\left(T_{i}^{-1}+T_{j}^{-1}\right)^{-1}\left(e_{i}+e_{j}\right)
$$

From the above equation its clear that a dipole whose ends points are $X_{1}$ and $X_{2}$ respectively, the center of mass is sensitive to which brane $i, j$ it ends. In fact the mass, $m_{i j}$, of the string connecting the two D6 branes, at $\vec{r}=\overrightarrow{r_{i}}$ and $\vec{r}=\overrightarrow{r_{j}}$, can be easily determined from our M-theory picture. It is given by the following integral over $\left(\vec{r}, x^{7}\right)$ space:

$$
m_{i j}=T_{M 2} \int_{S_{i j}} H_{0}^{\frac{1}{2}} H_{1}^{-\frac{1}{6}} H_{2}^{-\frac{1}{6}} H_{3}^{-\frac{1}{6}}|d \vec{r}| d x^{7}
$$

where $T_{M 2}$ is the tension of the M2 branes and $S_{i j}$ is the sphere build by shrinking the $\left(\vec{r}, x^{7}\right)$ torus at two points $\overrightarrow{r_{i}}$ and $\overrightarrow{r_{j}}$ as shown below:

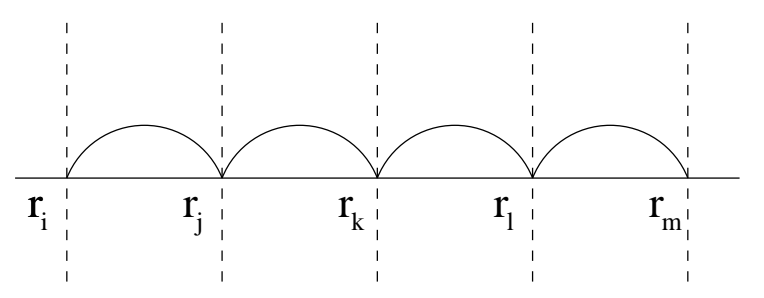

We will assume that the total mass of the string is distributed in a particular way when we go to the LSQM approach. The center of mass $X_{(c) i j}$ is therefore important. Though

5 We choose to denote the bases vectors as $e_{i}, e_{j}$ instead of $e_{1}, e_{2}$ because of the obvious indications from (eq. 4.13). We hope that this will not confuse the readers. 
its meaningful to write $X_{(c) i j} \equiv X_{i j}$, we will however use only $X_{c}$ to denote the center of mass. The full components will be used only where we need to specify the exact details of interactions.

The change of basis is now readily calculable and its given by

$$
\left\langle e_{i}^{a}, e_{j}^{a} \mid X_{c}\right\rangle=e^{-\imath\left(e_{i}^{a}-e_{j}^{a}\right)\left(\frac{1}{2}\left(T_{i}^{-1}+T_{j}^{-1}\right) X_{c}\right)^{A}} \delta\left(\frac{e_{i}^{a}+e_{j}^{a}}{2}-\frac{\left(\left(T_{i}^{-1}+T_{j}^{-1}\right) X_{c}\right)^{a}}{2}\right),
$$

where the equality is upto a normalisation.

Now its again straightforward to use (eq. 4.11). The result can be presented compactly as before [6]:

$$
\left(\Psi_{j}^{i} * \Phi_{k}^{j}\right)(X)=\left.\exp \left(\frac{\imath}{2} \frac{\partial}{\partial X^{\prime \mu}} \Omega_{i j ; j k} \frac{\partial}{\partial X^{\prime \prime \nu}}\right) \Psi_{j}^{i}\left(X^{\prime}\right) \Phi_{k}^{j}\left(X^{\prime \prime}\right)\right|^{X^{\prime}=S_{i j}^{i k} X, X^{\prime \prime}=S_{j k}^{i k} X}
$$

where $\Omega_{i j ; j k}$ can be given in terms of $T_{i}$ 's as:

$$
\Omega_{i j ; j k}=\left(\frac{T_{i}^{-1}+T_{j}^{-1}}{2}\right)^{-1} J\left(\frac{T_{j}^{-1}+T_{k}^{-1}}{2}\right)^{\top \cdot(-1)}
$$

The above equation is the precise mathematical formulation of non-Abelian geometry. The noncommutativity parameter $\Omega_{i j ; j k}$ is now sensitive to which brane the dipole end points lie. The quantity $J$ is the usual noncommutativity 6 (due to $B$ field) and $T_{i}$ 's are due to the non abelian nature. (eq. 4.25) therefore encodes this intertwining clearly and there is no way to separate them. In terms of string diagrams we need
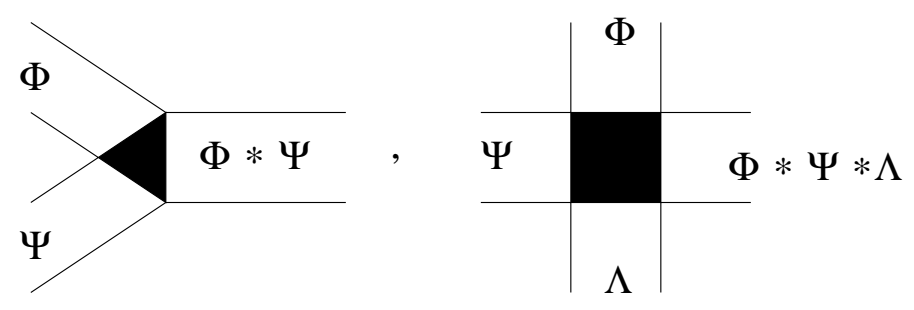

where the basic triangle vertex and the next square vertex will be used in the following section to understand some of the dynamics of the theory. These vertices are meant to simplify the evaluations of the nonabelian star product and have no other deeper significances.

6 Multiple noncommutativity on the branes were first discussed in [28, in connection with conifold geometry, and in [29], for parallel branes in a slowly varying background field. 


\section{Some Applications}

So far we have developed the formalism of deformed product with some relation to string theory. Even though the Taub-NUT setup has its roots in string theory, its not very obvious how to embed the nonabelian geometry in string theory. As discussed earlier, stability is an issue here. Therefore there are two interesting aspects of the problem: (a) non-trivial $H_{N S N S}$ background, and (b) supersymmetry. Since $H_{N S N S}$ contributes to the energy-momentum tensor we would need a curved background. For the case when the $H_{N S}$ field was oriented in such a way that it is constant along the D6 branes, the TN background in M-theory was sufficient to explain the dynamics. As we saw in great detail above, the M-theory way of looking at it has an advantage. It gives us an unified way to study many related theories which are generated by choosing (in string theory) various orientations of $B$ field along the brane [1,2, $5,6,6,10]$. The most generic background is of course the multiTN background with G-fluxes with or without M5 branes. Understanding the dynamics of this background will give more detailed account of all the theories mentioned and their product structure. Let us now study some applications of the various scenarios discussed in the earlier sections.

\subsection{Parallel Branes}

The information contained in $\Omega_{i j ; j k}$ can be represented in terms of dipole diagrams which can clarify the main idea of non-Abelian geometry. Let us consider N D-branes labelled by $i, j, k, \ldots$ and fix two points on branes $i$ and $k$. Then to calculate the deformed product we need to give weight to the triangle

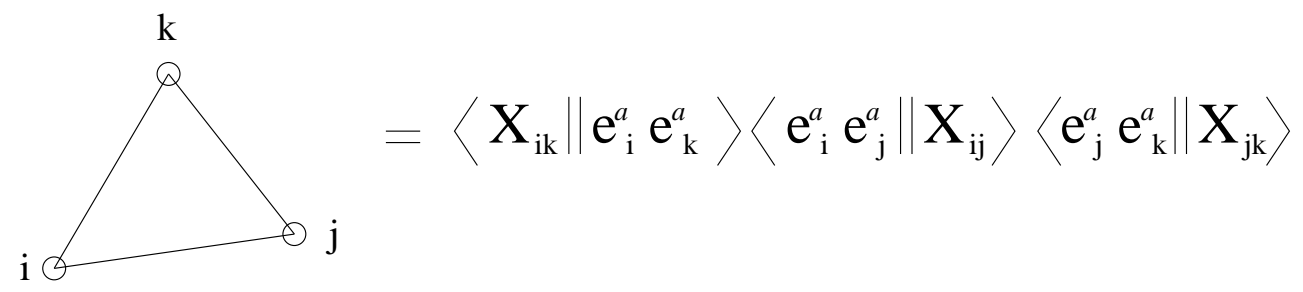

where $j$ is any arbitrary point. For the given points $i, k$ the orientation of the triangle is:

$$
\overrightarrow{i j}+\overrightarrow{j k}+\overrightarrow{k i}=0
$$

7 Observe that the two legs $i j$ and $j k$ of the triangle only provide spatial components from $\frac{D}{2}+1$ to $D$ or 1 to $\frac{D}{2}$. Therefore to get all the components we have to include the leg $i k$. This is also in some sense the root of the "cross" product. From (eq. 4.23) it is clear that $X_{c}^{a}$ are correlated to $X_{c}^{A}$ via the matrices $T_{i, j, k}$ and $J$ and vice-versa. There is of course no correlation between $a a$ and $A A$. Therefore the noncommutativity parameter comes from full triangle $i j k$. And since $j$ is summed over, all branes participate. 
Therefore the quadratic interactions are given by the following diagram:

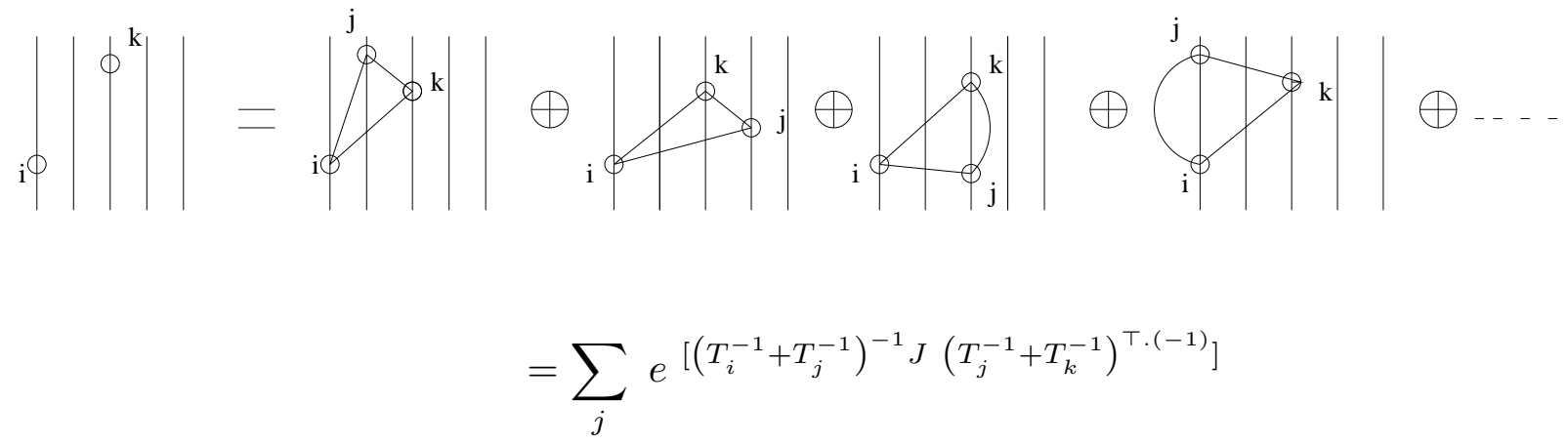

Let us now consider a case in which the wavefunctions are concentrated near one of the brane labelled by 1 . In terms of the above formula we now have 8

$$
\sum_{k=1}^{N} e^{\left[\left(1+U_{1 k}\right)^{-1} \Omega_{1}\left(1+U_{1 k}\right)^{\top \cdot(-1)}\right]}=e^{\left[\Omega_{1}\right]} \oplus \ldots
$$

where $U_{1 k}=T_{1} T_{k}^{-1}$.

This would imply that near one of the brane (say 1), the non-commutativity parameter is given by $\Omega_{1}$. This is consistent with naive expectation [29]. Here what we see that the non-abelian nature could in principle contribute to $\Omega_{1}$. However as we shall show in the next section, the matrix $U_{i j}$ depends upon the ratio of the two background fields $\mathcal{F}_{i, j}$. For small variations of the fields $U_{i j}$ 's are small. In terms of dipole diagrams we have
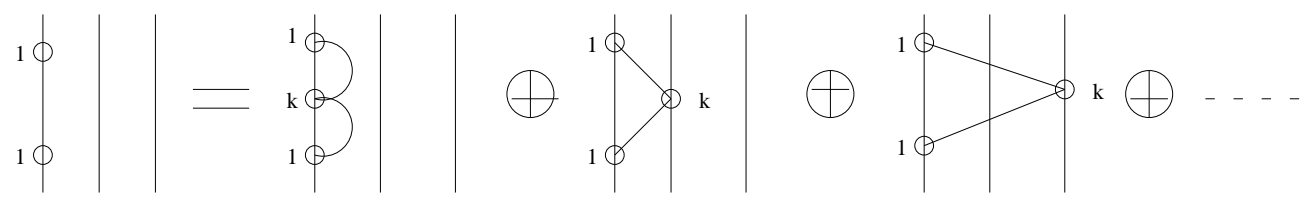

Observe that the first diagram contributes as $\Omega_{1}$.

Let us see what are the fundamental dipole diagrams if we go to higher order in interactions. Consider the following cubic interaction:

$$
(\Phi * \Psi * \Lambda)_{l}^{i}=\left(\Phi_{j}^{i} *_{i j k} \Psi_{k}^{j}\right) *_{i k l} \Lambda_{l}^{k} .
$$

Of course the above product is associative. Therefore it doesn't matter which way we multiply. It turns out that the explicit product involves the following terms:

$$
\Omega_{i j ; j k}, \quad \Omega_{j k ; k l}, \quad \Omega_{i j ; k l}
$$

8 By [ ] here we always mean that the terms are contracted by the corresponding momenta of the wavefunctions appearing alongwith. For details see [6]. We also neglect the numerical factors. 
In terms of dipole diagrams this is:

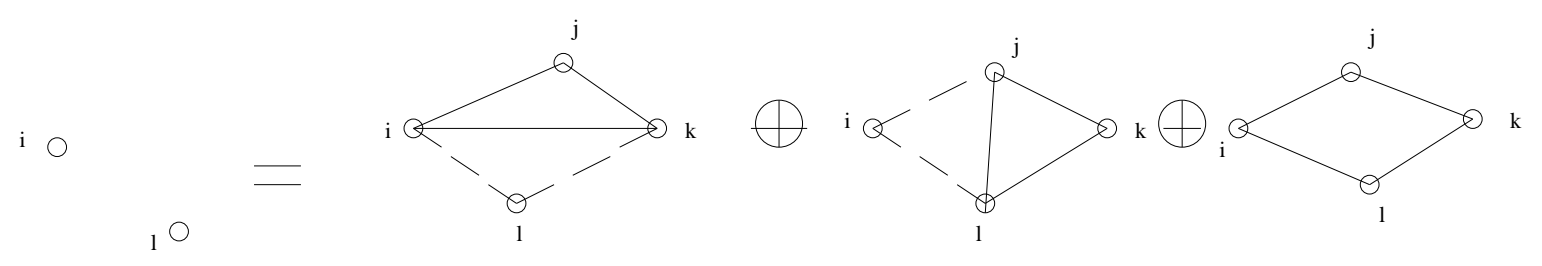

As is obvious from above knowing the triangle and the quartic we can predict all higher order interactions.

Another related observation is to have two branes located at the two opposite ends of a circle. For example let us have two parallel $D 3$ branes oriented along $X^{0,1, . .3}$ and are on a circle $x^{6}$. Let us also put gauge fields such that

$$
\mathcal{F}_{23}^{1}=\epsilon^{-\alpha}, \quad \mathcal{F}_{23}^{2}=\epsilon^{-\beta} .
$$

This model can be related to the $D 0-D 4$ system studied in [10] where the $D 4$ has a large amount of flux. For two D3 branes parallel to each other and having some flux the shift in the mode numbers

$$
\nu=\frac{1}{\pi}\left(\tan ^{-1} \mathcal{F}_{1}-\tan ^{-1} \mathcal{F}_{2}\right)=0 .
$$

(For equal and opposite fluxes the shift $\nu=1$ [6]). We now ask: what is the deformation product for this theory?

The above model can be represented by an infinite array of $D 3$ branes with noncommutativity parameters $\Omega_{1}, \Omega_{2}, \Omega_{1}, \ldots$

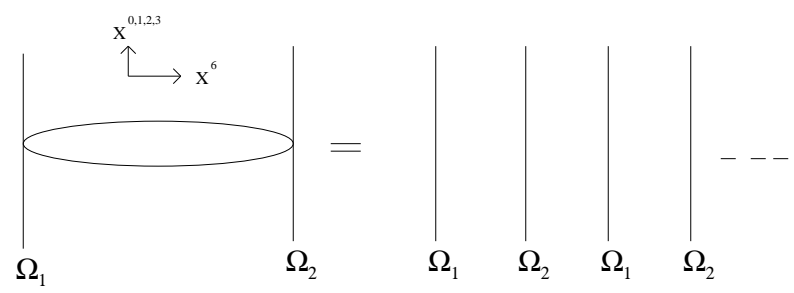

This would mean that the infinite array of dipole diagrams can now be represented by only two diagrams, implying, for example for the case $\alpha<\beta$,

$$
\sum_{k} e^{\left[\Omega_{1 k ; k 1}\right]}=e^{\left[\Omega_{1}\right]} \oplus e^{\left[4 \Omega_{2}\right]}
$$

where we have properly normalised the RHS. 


\subsection{Brane-Antibrane theory}

So far we have been concentrating on scalar fields. Let us now take a configuration of branes and antibranes stacked parallel and alternate to each other. Let us denote them by $i, j, k, .$. where $i$ is a brane, $j$ is an antibrane and so on. The $i i$ string will give vector multiplets (containing vectors $A_{\mu}$ and scalars $\left.\Phi\right)$ and so would the $j j$ string. But the strings $i j, j k, k l$ etcs. will each give rise to a complex tachyon: $\mathcal{T}_{i j}, \mathcal{T}_{j i}=\mathcal{T}_{i j}{ }^{*}$. Because of the tachyons this system is unstable and it breaks all supersymmetry.

For the moment let us not bother about the instability of the system. We shall return to this issue soon. Also for definiteness let us concentrate on a system of D5 and $\overline{D 5}$ labelled by 1 and 2 respectively. The five branes are oriented along $x^{0,1, . ., 5}$.

Along directions $x^{4,5}$ we switch on a gauge field $F_{i}$ and a $B$ field, such that on each brane we have $\mathcal{F}_{i}=F_{i}-B$. This will make the theory on each brane non-commutative. For later use let us define the following quantities:

$$
\mathcal{F}_{i}-\mathcal{F}_{j} \equiv \Delta, \quad \frac{1}{4}\left(\Omega_{i}-\Omega_{j}\right) \equiv \nabla
$$

Let us first consider the product: $(\mathcal{T} * \Phi)_{12}=\mathcal{T}_{12} * \Phi_{22}$. From our previous discussions this will be defined by

$$
\Omega_{12 ; 22}=\left(1+U_{21}\right)^{-1} \Omega_{2},
$$

where we have already defined the matrix $U_{i j}$. It turns out that the matrix $U_{i j}$ satisfy

$$
\Omega_{i}=U_{i j} \Omega_{j} U_{i j}^{\top}
$$

as well as the cocycle condition

$$
U_{i j} U_{j k}=U_{i k}
$$

The above two relations can be used, along with the fact that the center of mass coordinate $X_{c}$ 's commute among themselves, to rewrite $U_{i j}$ in terms of the background fields $\mathcal{F}_{i, j}$ as

$$
U_{i j}=\left(1+2 \Gamma \mathcal{F}_{i}\right)^{-1}\left(1-2 \Gamma \mathcal{F}_{j}\right)
$$

This is the relation we want for extracting more information from (eq. 5.7) . The matrix $\Gamma$ appearing in (eq. 5.10) can be related to $\Delta$ and $\nabla$ defined earlier via the following relation:

$$
\Gamma^{\top}-\Gamma+\Gamma \Delta \Gamma^{\top}=\nabla
$$

The above relation exists as one can easily show that $\nabla \Delta$ can never have eigenvalue 1 . 
Before going any further we need to see how the tachyons behave in this system. For this let us study the system of $D 5-\bar{D} 5$ with fluxes on its world-volume in some details 9 . The reason why we took a system of $D 5-\bar{D} 5$ is because this configuration can be related to the case of a $D 5-\bar{D} 5$ brane wrapped on a vanishing two cycle of a conifold [28]. There is a non trivial $B$ flux on the two cycle, and since the two cycle is of vanishing size the $B$ field is actually infinite. Similarly there is also a gauge flux on the brane. It was conjectured in [31] that for some special choice of the background fields the system is supersymmetric and the tachyon is massless. This was eventually shown to be the case in [28].

The quantization of open strings connecting a brane to an antibrane is well-known. In the NSR formalism, it is identical to the usual quantization of open strings except that the GSO projection is opposite to the usual one. Hence we keep the "anti-GSO" states. These include, at the lowest levels, a tachyon of $M^{2}=-\frac{1}{2}$. In addition we find a set of massless fermions which are obtained by dimensionally reducing a single 10-dimensional Majorana-Weyl fermion, of opposite chirality to the usual GSO-projected one, down to $p+1$ dimensions.

In the case of interest here, this quantization is modified for two reasons. First, there is a constant B-field, experienced by both the brane and the antibrane, and also a worldvolume field strength $F$ on one of the pair. Second, the brane and antibrane are both wrapped around a 2-cycle of vanishing size.

Let us work out the quantization of open strings joining a D5-brane to a $\overline{\mathrm{D}} 5$-brane in the presence of fluxes. Let $b_{1}=\left(F_{1}-B\right)$ and $b_{2}=\left(F_{2}-B\right)$, where $F_{i}$ are the worldvolume gauge fields on the $i$ th brane and $B$ is the constant spacetime $B$-field. Also, let $z=x^{4}+i x^{5}$. We have chosen to allow nonzero $F$ and $B$ values only along the two directions $x^{4,5}$.

The boundary conditions are:

$$
\begin{aligned}
& \left(\partial_{\sigma} z+b_{1} \partial_{t} z\right)_{\sigma=0}=0 \\
& \left(\partial_{\sigma} z+b_{2} \partial_{t} z\right)_{\sigma=\pi}=0
\end{aligned}
$$

and a similar condition for $\bar{z}$ ( with $b_{i} \leftrightarrow-b_{i}$ ).

Let us now write the mode expansion as:

$$
z=\sum_{n} A_{n+\nu} e^{(n+\nu)(t+i \sigma)}+\sum_{n} B_{n+\nu} e^{(n+\nu)(t-i \sigma)} .
$$

9 A somewhat similar behavior of tachyons were studied in a different context for a system of a D3 parallel to a D7 in the presence of non-primitive fluxes in 30]. 
The first boundary condition yields

$$
A_{n+\nu}=B_{n+\nu} \frac{1+i b_{1}}{1-i b_{1}}
$$

while the second one gives

$$
e^{2 \pi i \nu}=\frac{\left(1-i b_{1}\right)\left(1+i b_{2}\right)}{\left(1+i b_{1}\right)\left(1-i b_{2}\right)} .
$$

Now recall that the 2-cycle on which $b_{i}$ are valued is of zero size, which is the same as saying that the value of the field $b_{i}$ is infinite, to keep constant flux. Thus we really need the above formula for infinite $b_{1}, b_{2}$, except that they can each be separately positive or negative infinity. Solving the above, one finds:

$$
\nu=\frac{1}{2 \pi}\left(-\tan ^{-1} \frac{2 b_{1}}{1-b_{1}^{2}}+\tan ^{-1} \frac{2 b_{2}}{1-b_{2}^{2}}\right)
$$

Now the relevant values are:

$$
\begin{aligned}
& \lim _{b \rightarrow-\infty} \tan ^{-1} \frac{2 b}{1-b^{2}}=0, \\
& \lim _{b \rightarrow 0} \tan ^{-1} \frac{2 b}{1-b^{2}}=\pi, \\
& \lim _{b \rightarrow \infty} \tan ^{-1} \frac{2 b}{1-b^{2}}=2 \pi .
\end{aligned}
$$

These formulae are analogous to similar results in Ref. 10 for the D0-D4 system. It is interesting to compare the two at this stage. In the latter case, there are altogether two pairs of directions over which a flux is allowed, while we have only one. Also, in that problem there are DN strings even in the absence of flux because the two branes have different dimensions. While Ref. [10] finds that a flux can make a tachyon appear in a system that was BPS before, we will instead find that a flux can make a tachyonic braneantibrane system into a BPS configuration.

Returning to the formula (eq. 5.16), we can use (eq. 5.17) to evaluate it for the relevant possibilities $\left(\left|b_{i}\right|=0, \infty\right.$ for each $\left.i\right)$. The result is easily seen to be

$$
\nu=\frac{1}{2}\left(\operatorname{sign}\left(b_{2}\right)-\operatorname{sign}\left(b_{1}\right)\right)
$$

where $\operatorname{sign}\left(b_{i}\right)=0, \pm 1$.

Note that for our purposes, $b_{1}=F_{1}-B, b_{2}=F_{2}-B$. Hence the case which we expect to be BPS comes about when $F_{1}=0$, so that $\operatorname{sign}\left(b_{1}\right)=-1$ while $\operatorname{sign}\left(b_{2}\right)=1$, 
and $\nu=1$. On the other hand, with $F_{1}=F_{2}=0$ we would find $\nu=0$ and this is the case where we do expect a tachyon.

It only remains to find out the zero-point energy as a function of $\nu$, in the NS sector (which is where the tachyon appeared, in the absence of flux). We use:

$$
\sum_{n \geq 0}(n+\nu)=-\frac{1}{12}\left(6 \nu^{2}-6 \nu+1\right) .
$$

The bosons along direction $x^{4,5}$ are quantised with mode numbers $n+\nu$ and the fermions have mode numbers $n \pm\left|\nu-\frac{1}{2}\right|$. Thus the zero point energy of the system will be

$$
E=2 E(0)+E(\nu)+2 E(0)-E(0)-3 E(1 / 2)-E(|\nu-1 / 2|) .
$$

The first term comes from $x^{0,1,2,3}$, the second from $x^{4,5}$, the third from $x^{6,7,8,9}$ and the fourth from the bosonic ghosts. The remaining terms are fermionic contributions. Adding up all the contributions, we get:

$$
E=-\frac{1}{2}\left(\left|\nu-\frac{1}{2}\right|+\frac{1}{2}\right)
$$

The case of no fluxes is $\nu=0$ while the case of fluxes relevant to fractional branes, as we argued above, is $\nu=1$. From the above formula we seem to find that both $\nu=0$ and $\nu=1$, the ground-state energy is $E=-\frac{1}{2}$ and hence there is a tachyon.

However the actual result is more subtle because of the GSO projection 10 . At zero flux, along with the open string tachyon there is always a massless state created by a world-sheet fermion (in the NS sector) $\psi_{-\frac{1}{2}}$. This is in fact a spacetime scalar or vector (depending on whether the fermion mode has an index transverse to the brane or along the brane). Now when there is flux, this mode (for the directions along which the flux is present) becomes $\psi_{-\left|\nu-\frac{1}{2}\right|}$. Thus the corresponding state has energy

$$
E=-\frac{1}{2}\left(\left|\nu-\frac{1}{2}\right|+\frac{1}{2}\right)+\left|\nu-\frac{1}{2}\right|=\frac{1}{2}\left(\left|\nu-\frac{1}{2}\right|-\frac{1}{2}\right) .
$$

Thus altogether we have a pair of low-lying states, one of energy $-\frac{1}{2}\left(\left|\nu-\frac{1}{2}\right|+\frac{1}{2}\right)$ and the other of energy $\frac{1}{2}\left(\left|\nu-\frac{1}{2}\right|-\frac{1}{2}\right)$. At $\nu=0$ these states have energies $-\frac{1}{2}, 0$ respectively, and

10 The discussion in the next two paragraphs were explained to us by Sunil Mukhi. We thank him for a detailed discussion of the tachyonic behaviour of this model. See also [32] for a somewhat related system. 
at $\nu=1$ they also have energies $-\frac{1}{2}, 0$. But if we tune $\nu$ continuously from 0 to 1 , we find that at $\nu=\frac{1}{2}$ the states become degenerate in energy, with both having $E=-\frac{1}{4}$. It turns out that at this point the two states cross each other.

To see this more explicitly, observe that the energies of the pair of states can equivalently be written $-\frac{1}{2} \nu$ and $\frac{1}{2}(1-\nu)$ for all $\nu$, without any $\bmod$ sign. In this way of writing it, the energies vary smoothly with $\nu$. These expressions, and not the earlier ones involving modulus signs, are the correct ones if we want to follow the evolution of a given state (with a given sign under GSO projection) as $\nu$ varies. Now we see that the tachyon at $\nu=0$ becomes massless at $\nu=1$. On the other hand the massless state at $\nu=0$ becomes tachyonic at $\nu=1$. But since we are in a sector with anti-GSO projection, the latter state is projected out for any $\nu$ ! The physical (anti-GSO) state, which is tachyonic at $\nu=0$ really does become massless at $\nu=1$. Thus we have shown that the tachyon disappears in the presence of flux, as desired. In the figure below we sketch the behavior of the tachyon for this system:

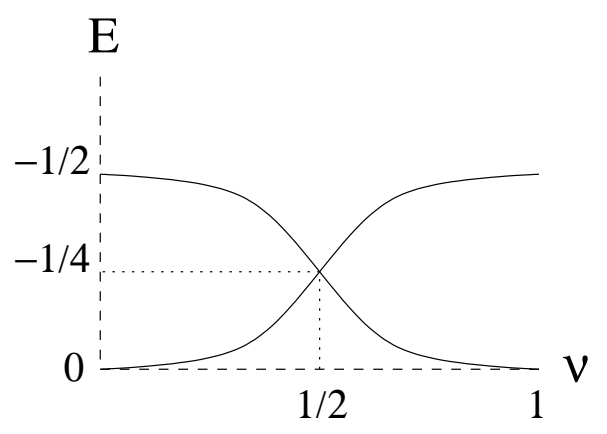

There are two subtleties in the problem which we shall be ignoring. First, the $B$ and the $F$ fields on the two cycle are not constant so we cannot actually use the whole arguments of non-abelian geometry. But we shall take a simplified case in which the fields are actually constant. We will return to the issue on nonconstant fields on the brane in the next section. The second subtlety has to do with the spherical cycle. We shall take the brane and the antibrane to be wrapped on a toroidal cycle. For vanishing cycle we will take the limit in which the fields approach infinity such that their ratio is finite. Therefore, for finite size of the cycle, $U_{21}$ is finite and small and $\Omega_{12 ; 22}=\Omega_{2}+\mathcal{O}(U)$. Thus the multiplication rule is

$$
\mathcal{T}_{12} * \Phi_{22}=\mathcal{T}_{12} *_{2} \Phi_{22}+\mathcal{O}(U)
$$

Since both $\mathcal{F}_{1} / \mathcal{F}_{2}$ and $\mathcal{F}_{2} / \mathcal{F}_{1}$ can be made finite, $U_{12}$ is also finite. This in turn implies:

$$
\mathcal{T}_{21} * \Phi_{11}=\mathcal{T}_{21} *_{1} \Phi_{11}+\mathcal{O}(U)
$$


Question now is what should be $\mathcal{T}_{12} * \mathcal{T}_{21}$ ? Here it would seem that either of $*_{1}$ or $*_{2}$ could suffice. To fix this let us go to higher order interactions: $(\mathcal{T} * \Phi * \mathcal{T})_{11}=\mathcal{T}_{12} * \Phi_{22} * \mathcal{T}_{21}$. From the dipole diagrams in the previous section we have seen that this is uniformally generated by:

$$
\begin{aligned}
{\left[\Omega_{12 ; 22}\right] \oplus\left[\Omega_{22 ; 21}\right] \oplus\left[\Omega_{12 ; 21}\right]=} & {\left[\left(1+U_{21}\right)^{-1} \Omega_{2}\right] \oplus\left[\Omega_{2}\left(1+U_{21}\right)^{\top \cdot(-1)}\right] } \\
& \oplus\left[\left(1+U_{21}\right)^{-1} \Omega_{2}\left(1+U_{21}\right)^{\top .(-1)}\right]
\end{aligned}
$$

implying that the product rule here could possibly be $\mathcal{T}_{12} *_{2} \mathcal{T}_{21}$. And similar for other cases. The dipole diagrams are:
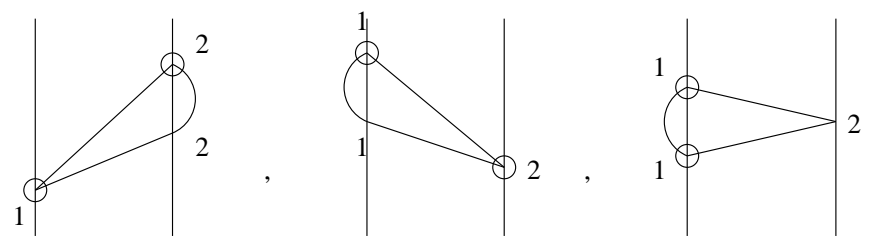

There could be other ways of writing the deformation product between the fields but the above procedure tells us that a consistent approximation is 1

$$
\Psi_{i j} *_{i j k} \Lambda_{j k}=\Psi_{i j} *_{j} \Lambda_{j k}+\mathcal{O}(U)
$$

for the specific case of this problem. $\Psi$ and $\Lambda$ could be $\mathcal{T}, \Phi$ or gauge fields. Thus we see that (eq. 4.24) reproduces all the known results and also shows what are the possible corrections. These corrections are basically because of the intertwining of the usual *product and the non-abelian space.

These results can be easily extended for the case of nonbps branes. Consider two $D 6$ branes in type IIB theory. These are unstable branes and there are now four tachyons in the system. In the presence of $\mathcal{F}_{i}$ fields on the branes one can use (eq. 4.24) to determine the product rules for multiplying tachyons in this theory.

\subsection{Branes with $H_{N S N S}$}

So far we have considered the case when the $G$ fluxes were decomposed over the ASD harmonic two form $\omega$ in such a way that the components along the D6 brane(s) are constant. We can now consider the case when the $G$ flux is a very generic function of space coordinates. From D6 brane point of view there are two interesting cases now:

11 Since the triangle has orientation, the apparent ambiguity arising due to complex conjugation isn't there. 
(a) There is a non-trivial $H_{N S}$ field on the world-volume of D6.

(b) There is a non-trivial $H_{N S}$ vanishing at the brane location.

Both these cases can actually arise in the hybrid theory setup that we described earlier. However we have to be a little careful now. The two D6 branes are oriented along $x^{0,1,2, \ldots, 6}$ and along $x^{0,1, \ldots, 4,8,9}$. In the hybrid theory that we describe we have, among other threeform fields in type IIA theory, the $B$ field $B_{16}$ which have both its legs on one D6 and only one leg along the other D6. This gives us a hint to use the same framework for the case when we have a $B$ field which is (say) $B_{89}$. In terms of M-theory we need a threeform flux $C_{789}$ in the conifold background. The above choice of $B$ field immediately tells us that we have a pinned brane like scenario on the other D6 (because the $B_{89}$ field is completely orthogonal to the D6). In the pinned brane scenario we saw in the earlier sections that the hypermultiplets pick up masses, and the mass is determined by the asymptotic value of fluxes. What would this imply for the other brane? On the other brane we see a $B$ field with both legs on the brane. But some of the scalars that have become massive due to the first brane continue to be massive on the other brane also. Furthermore since the $B$ field would vary non-trivially on the orthogonal directions of the first brane as a result we will see a non-constant $B$ field on the other D6 brane, i.e a $H_{N S}$ field! Thus the conifold like geometry that we discussed in earlier sections can in fact also allow us to study this scenario.

Question now is whether we could say something about any kind of star product here? For the hybrid brane scenario, when we have non-zero $H_{N S}$ on the other D6 brane, the open string fluctuations are goverened by an additional term on the world-sheet $\Sigma$ (discussed also in a slightly different context in [33]):

$$
\frac{i}{3 \pi \alpha^{\prime}} \int_{\Sigma} H_{a b c} X^{a} d X^{b} \wedge d X^{c}
$$

where $H_{a b c}$ is the threefrom on the $\mathrm{D} 6$ brane. There are of course the usual terms on the world-sheet that we ignore here. This theory also have a star product governed by (in some limits) the inverse of $\widetilde{\mathcal{F}}_{a b} \equiv \mathcal{F}_{a b}+\frac{1}{3} H_{a b c} x^{c}$, where $\mathcal{F}_{a b}$ is used in the previous sections to evaluate the Moyal product. If we ignore, for the time being the other D6 brane giving rise to the pinned brane scenario, we would have a similar TN background with fluxes. However the G-fluxes now needed is not the one discussed in (eq. 4.19) but rather a more involved one as

$$
\frac{G}{2 \pi}=\sum_{i=1}^{N} \mathcal{F}_{i} \wedge \omega_{i}+d z \wedge \gamma_{1}+d \bar{z} \wedge \gamma_{2}
$$


where $z, \bar{z}$ are one-forms in this background (with some specific choice of complex structure 12 and $\gamma_{1,2}$ as the three-forms that eventually determine $\widetilde{\mathcal{F}}$. This choice of G-flux is now used to determine the star product for the system following the analysis presented in [13]. It turns out that this star product is in fact non-associative and the non-associativity is given by (first shown in [33]):

$$
(\Phi \star \Psi) \star \Lambda-\Phi \star(\Psi \star \Lambda)=\left(\gamma_{1}+\gamma_{2}\right)_{a b c} \lambda^{a b c}
$$

where $\lambda^{a b c}$ is in general non-zero and is a function of the fields $\Phi, \Psi, \Lambda$ as discussed in [33]. The non-associative interaction is evident directly from the BI action of the D6 brane written in terms of $\sqrt{\operatorname{det}(g+\gamma \cdot x+. .)}$ where $\gamma=\gamma_{1}+\gamma_{2}$ and $x$ is the coordinate on D6. Notice also that we haven't put any conditions on $\gamma_{1,2}$. Supersymmetry would require the minimal constraint: $\gamma_{2}=* \gamma_{1}$. However the fact that the algebra is non-associative hinders any nice description of the system.

This situation could improve if we consider the case when the $H$ field vanishes at the point where one of the D6 is placed 13 . Therefore a supersymmetic configuration with the choice of G-flux (eq. 5.28) is now further constrained. The generic behavior, in the absence of field strength, is that $\gamma_{1}$ should at least be anti self-dual. This ASD property changes to

$$
* \gamma_{1}=-\gamma_{1}+\sum_{n} a_{n} r^{n}
$$

where $r$ is the coordinate orthogonal to the D6 brane. Because of the other D6 brane which is pinned to the origin $r=0$, our D6 brane will also remain fixed at the origin and therefore we might naively expect the ASD property to be violated as $* \gamma_{1}=-\gamma_{1}+a_{0}$. Making $a_{0}=0$ will give an exact ASD condition on $\gamma_{1}$ such that the background three-form on the brane $\gamma_{1}+\gamma_{2}=0$. This would also mean that (eq. 5.29) is no longer the case and the algebra will tend to be associative even though we have a non zero three-form parallel to the brane. Observe that the pinning of the branes in this case is very important. If we allow the un-pinning effect in the conifold setup, then probably associativity for this case may be broken. This needs to be verified.

There is yet another extension of the above discussion that could in principle arise in the hybrid theory. Till now we saw two interesting cases: (a) Dipole plus noncommutative

12 These one forms parametrise a $T^{2}$ in the conifold geometry. This is the same $T^{2}$ on which by making two T-dualities we could get a brane box configuration in type IIB theory.

13 A similar configuration is recently been shown to occur (with D3 replacing the D6) in a parallellizable plane wave background [34]. It will be interesting to find a connection between the two scenarios. 
theory, and (b) Pinned brane theory plus noncommutative theory. We could extend this scenario further by taking the case of rank six $B$ field on one of the D6 brane so that we have (for example) the following components: $B_{12}, B_{34}, B_{89}$. For the other D6 oriented along $x^{0,1, \ldots, 6}$ there is a noncommutative algebra along directions $x^{0,1,2,3,4}$ but also some possibility of pinning due to $B_{89}$ (whether or not there is a real pinning needs to be verified by sugra analysis). On the other hand the second D6 sees a complete noncommutative algebra now. We are assuming for simplicity that there is no $H$ field and also all the $B$ fields have equal magnitudes. Viewing now the conifold as an ALE fibration over a constant slice of the $P^{1}$ base the value of $g(r)$ can be estimated for this case using the generic rule given in (eq. 2.3) . The result is 14

$$
g(r)=\frac{r(1+\sec \alpha)^{2} \exp \left(2 \sqrt{\frac{2 r+R \sec ^{3} \alpha}{2 r \sec ^{2} \alpha+R \sec ^{3} \alpha}}-\frac{2}{\sec \alpha}\right)}{\left(1+\sec ^{2} \alpha\right) r+\sec ^{3} \alpha R+\sqrt{\left(2 r \sec ^{2} \alpha+R \sec ^{3} \alpha\right)\left(2 r+R \sec ^{3} \alpha\right)}} .
$$

In deriving this we have ignored the effect of stuck D0 branes on the D6 brane as mentioned in [13]. Taking the other slice of the conifold we have a rank four $B$ field on the D6 with an additional $B$ field orthogonal to it. The detailed dynamics of this system is complicated which we hope to tackle in near future.

\subsection{Theory near multi-Conifold type geometry}

So far we have discussed examples with single centered Taub-NUT geometry and multi centered TN geometry. We also saw that a $P^{1}$ fibration of the TN or ALE space gives rise to a conifold type geometry. Question now is to understand what happens if we have the multi TN fibered over a $P^{1}$ base. The family of resolved ALE spaces with $A_{n-1}$ singularities is given by the equation

$$
z_{1}^{2}+z_{2}^{2}+\prod_{i=1}^{n}\left(z_{3}-\mu_{i}\right)=0
$$

where $z_{i}$ are the coordinates of a $C^{3}$ and the $S^{2}$ mentioned in the earlier sections are related to $\mu_{i}$ as $S_{i j} \leftrightarrow \mu_{i}-\mu_{j}$. The full $A_{n-1}$ symmetry is realised when all the $S^{2}$ shrink to zero size. From here it is now clear that the multi conifold geometry can be realised when we consider the following fibration

$$
z_{1}^{2}+z_{2}^{2}+\prod_{i=1}^{n}\left(z_{3}^{2}+z_{4}^{2}-\mu_{i}\right)=0
$$

14 Done in collaboration with Govindan Rajesh. 
Over the curve $z_{1}=z_{2}=0$ we have the ALE $A_{n-1}$ transversal fiber. The above consideration opens up yet another set of theories that could be studied in M-theory on a multi conifold type of background in the presence of fluxes and probed by a M5 brane background.

These singularities which have been discussed earlier in [20], can actually be related to the quotient of a conifold for the case when $\mu_{i}=0$. This quotienting is a simple $\mathbf{Z}_{n}$ action [18] defined on the equation for the conifold written as

$$
X Y=Z^{n} W^{n}
$$

where $X, Y, Z, W$ can be easily related to $z_{i}$ of (eq. 5.33). We could even extend this further by taking the quotient operation as $\mathbf{Z}_{m} \times \mathbf{Z}_{n}$. This geometry has also been discussed in [20] and takes the form

$$
X^{m} Y^{m}=Z^{n} W^{n}
$$

where we have again used the same coordinates. All these singularities are of course related to ALE fibration over some base. However we could go beyond that by taking a singularity for which powers of $Z, W$ are different 15 . This is clearly not related to any quotient of a conifold and might fall slightly off the main line of thought that we are pursuing here. Nevertheless it will be interesting to see any such connection.

All these M-theory configurations can be studied in type IIA also. In the type IIA side, as before, there are two possibilities. We could either come down via $x^{1}$ or via $x^{7}$ - the conifold cycle. The first case is simply the reduced conifold geometry whereas the second case is a set of $n$ orthogonal D6 branes with fluxes on its world volume. The hybrid theory discussion that we had earlier will now be more involved because we could now have a situation where the noncommutative geometry on one set of parallel D6 branes could be converted to a nonabelian geometry! Clearly now the star product is more involved because of this. But thats not the end of the story. We saw in the conifold setup that we could have dipole theory on the other brane. When we allow different fluxes on different D6 then there is a possibility to have different dipole lengths on the world volumes. The dipole star product will now have to mix somehow with the nonabelian nature of the D6 branes. This theory has never been studied before. It will be interesting to see whether a stable configuration can be realised in this setup.

15 For example manifolds of the form $X Y=Z^{m} W^{n}$. As discussed in [18 these manifolds can actually be generated in type IIB theory from the T-dual picture where we have intersecting set of NS5 branes. Putting $m$ number of NS5 branes along one direction and $n$ number along the orthogonal direction can in fact generate this kind of singularity via one T-duality. 
Before we end this section we should point out that this geometrical construction can be extended even further by including the $D_{n}$ and $E_{n}$ kind of singularities. First let us look back again at the TN scenario. We discussed the following structures:

\section{$\mathrm{TN} \rightarrow$ Multi TN.}

We will also consider this from ten dimensional perspective. The above singularity is just the $A_{n}$ series of Kleinian singularities discussed in the literature. The TN that we discussed earlier was oriented along $x^{7,8,9,10}$ (with $x^{1}$ as the M-theory circle). Combining these to form $z^{1}, z^{2}$ in the obvious way, the $A_{n}$ singularities are generated by action $\Gamma$ on $C^{2} / \Gamma$ with the action being via a matrix with diagonal entries $e^{ \pm \frac{2 i \pi}{n+1}}$. This matrix, and together with an off-diagonal matrix with entries $i$ will now generate the $D_{n}$ singularities. From Mtheory point of view the wrapped M2 branes should now reproduce the intersection matrix of $D_{n}$ kind of singularities. Such a scenario is possible when we have a Atiyah-Hitchin kind of singularity on top of a TN singularity, at least locally. Analysis of this background in the presence of fluxes is subtle and a fully compact example (with all local charges cancelled) leads us to manifolds that are generically non-Kähler [35]. Section 2.6 of [35] discusses the full implications of a $D_{4}$ singularity and therefore we refer the reader to that. The $E_{n}$ singularities now fall under the binary tetrahedral, Octahedral and Icosahedral groups. The adjoining matrices in the three cases are given by

$$
\begin{array}{rlr}
T=\frac{1}{\sqrt{2}}\left(\begin{array}{cc}
\epsilon^{7} & \epsilon^{7} \\
\epsilon^{5} & \epsilon
\end{array}\right), & O=\left(\begin{array}{ll}
\epsilon & 0 \\
0 & \epsilon
\end{array}\right) \\
I_{1}=\left(\begin{array}{cc}
-\eta^{3} & 0 \\
0 & -\eta^{2}
\end{array}\right), & I_{2}=\frac{1}{\eta^{2}-\eta^{3}}\left(\begin{array}{cc}
\eta+\eta^{4} & 1 \\
1 & -\left(\eta+\eta^{4}\right)
\end{array}\right)
\end{array}
$$

where $\epsilon$ is the primitive eighth root of unity and $\eta$ is a fifth root of unity. The tetrahedral group is generated by $T$ and the octahedral group is generated by taking the elements of $T$ and adjoining them with the elements of $O$. The icosahedral group is generated by $I_{1}$ and $I_{2}$.

However this is not enough. There is still more to the above classification. It turns out that the above set of singularities are related to the chiral ground rings of a $c=1$ string theory at the self-dual radius [36,37]. An immediate question would be: what about the non-chiral rings? As discussed in [37], the non-chiral rings are responsible for the other kind of structures that we studied here, namely

\section{Conifold $\rightarrow$ Multi Conifold.}


The above structure is nothing but the $A_{n}$ series for the non-chiral ring. Therefore one would ask about the other $A-D-E$ singularities. Clearly many things can be said now, but we will not pursue this here anymore and leave the rest of the discussions for future work. The connection of our work to $c=1$ string theory provides yet another unified picture to view the whole dynamics.

\section{Discussions}

In this paper we followed two unified themes. First, there is a unification at the level of underlying gravitational solution. We start with a Taub-NUT space with background three-from fluxes in M-theory. When this background is fibered over a $P^{1}$ in some specific way we get a conifold like geometry with fluxes. This gives us a way to interpolate between two different backgrounds. Furthermore the Taub-NUT space could be made multi-centered. Fibering this over a $P^{1}$ now gives us a multi-conifold like geometry. Therefore in terms of geometry we have the following interpolating scenarios:

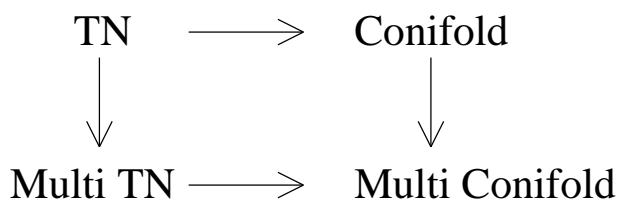

Secondly, there is a unification at the level of theories probing these backgrounds. All these theories appear in M-theory from a configuration of branes, fluxes and geometry. In fact the geometry (with fluxes) by themselves is responsible to generate either noncommutative, nonabelian, or even hybrid theory depending on specific configuration. When M5 branes probe this background, pinned brane theory or dipole theory can be studied. This aspect is interesting because many different theories are now different guise of one unified framework in M-theory. We also pointed out that the above scenario could be extended even further if we allow non-trivial $H_{N S}$ fields on the branes. A simple analysis showed that the star product becomes non-associative and therefore this would not be physically relevant. However when the $H_{N S}$ field vanishes on the brane (but no where else) then many interesting things can be said.

All the above theories studied are static in nature. We could ask whether it is also possible to study theories that have some inherent time dependences. Some aspect of this have been dealt recently in [38,13] 16 . Defining $x^{ \pm}$in the usual way, the metric in M-theory

16 In 38] explicit holographic dual for a time dependent closed string background was presented. The noncommutativity parameter was found to be non constant and the theory on the brane was shown to be decoupled from the bulk. For our case here, since we have taken D6 branes, the theory however is never decoupled. 
is defined in terms of two harmonic functions $H_{1,2}$, such that they are related by

$$
H_{1}-H_{2}=\left(\frac{x^{+}}{R_{o}}\right)^{2},
$$

where $R_{o}$ is the radius of the TN (the relation between $R_{o}$ and $R$ mentioned in this paper is related as $R=g_{s} l_{s}^{2} / R_{o}$ ). The explicit derivation of the above formula (eq. 6.1) is given in [13] and so we refer the reader to that for further details. To progress further along the generic idea presented in this paper, we need the value of $g(r)$. This can be derived using the generic rule given in (eq. 2.3) and the steps mentioned in [13] assuming the two form $\omega$ to be normalisable and harmonic for a fixed $x^{+}$. In terms of the variables defined above this is given by:

$$
g(r)=\left(1+\sqrt{1+\left(\frac{x^{+}}{R_{o}}\right)^{2}}\right)^{2} \frac{2 r}{\left(\sqrt{R+2 r}+\sqrt{R+2 r\left(1+\left(\frac{x^{+}}{R_{o}}\right)^{2}\right)}\right)^{2}},
$$

where we have again measured $g(r)$ wrt $2 \pi l_{p}^{2}$. Some aspects of this time dependent background has been addressed in [13] using the arguments of duality chasing. Since this example also falls in the description that we have given, broadens the horizon of the application of our general framework to time dependent cases also. It will now be interesting to see whether we could say something more about the conifold, multi TN and its generalisations from here. Clearly we have just scratched the surface and many more details need to be filled in to get a deeper understanding of the dynamics of oriented $B$-fields. We hope to address this in near future.

\section{Acknowledgements}

We would like to thank Sunil Mukhi for an explanation of the tachyonic behaviour of a brane anti-brane system and Govindan Rajesh for helping us calculate some of the properties of a Taub-NUT geometry. We would also like to acknowledge useful conversations with Ori Ganor, Daniel Robbins, M. M. Sheikh-Jabbari, Savdeep Sethi, and Zheng Yin on various aspects of these theories. The work of K.D is supported in part by a David and Lucile Packard Foundation Fellowship 2000-13856. The work of M.S is supported in part by DOE grant number DE-AC03-76SF00515. 


\section{References}

[1] S. Chakravarty, K. Dasgupta, O.J. Ganor and G. Rajesh, "Pinned Branes and New Non-Lorentz Invariant Theories," Nucl.Phys. B587 (2000) 228, hep-th/ 0002175.

[2] A. Bergman and O. J. Ganor, "Dipoles, twists and noncommutative gauge theory," JHEP 0010, 018 (2000), hep-th/0008030.

[3] A. Hashimoto and N. Itzhaki, "Non-commutative Yang-Mills and the AdS/CFT correspondence," Phys. Lett. B 465, 142 (1999), hep-th/9907166; J. M. Maldacena and J. G. Russo, "Large $N$ limit of non-commutative gauge theories," JHEP 9909, 025 (1999), hep-th/9908134.

[4] A. Bergman, K. Dasgupta, O. J. Ganor, J. L. Karczmarek and G. Rajesh, "Nonlocal field theories and their gravity duals," Phys. Rev. D 65, 066005 (2002), hepth/0103090.

[5] K. Dasgupta, O. J. Ganor and G. Rajesh, "Vector Deformations of $\mathcal{N}=4$ Yang-Mills Theory, Pinned Branes and Arched Strings," hep-th/0010072.

[6] K. Dasgupta and Z. Yin, "Non-Abelian Geometry", Commun. Math. Phys. 235, 313 (2003), hep-th/0011034.

[7] K. Dasgupta and M. M. Sheikh-Jabbari, "Noncommutative dipole field theories," JHEP 0202, 002 (2002), hep-th/0112064.

[8] M.R. Douglas and C. Hull, "D-branes and the Noncommutative Torus," JHEP 9802 (1998) 008, hep-th/9711165.

[9] N. Nekrasov and A. Schwarz, "Instantons on noncommutative $R^{* *} 4$ and $(2,0)$ superconformal six dimensional theory," Commun. Math. Phys. 198, 689 (1998), hepth/9802068.

[10] N. Seiberg and E. Witten, "String Theory and Noncommutative Geometry," JHEP 9909, 032 (1999, hep-th/9908142.

[11] M. M. Sheikh-Jabbari, "More on mixed boundary conditions and D-branes bound states," Phys. Lett. B 425, 48 (1998), hep-th/9712199; "Open strings in a B-field background as electric dipoles," Phys. Lett. B 455, 129 (1999), hep-th/9901080; D. Bigatti and L. Susskind, "Magnetic fields, branes and noncommutative geometry," Phys. Rev. D 62, 066004 (2000), hep-th/9908056.

[12] Z. Yin, "A Note on Space Noncommutativity," Phys. Lett. B466 (1999) 234, hepth/9908152.

[13] K. Dasgupta, G. Rajesh, D. Robbins and S. Sethi, "Time-dependent warping, fluxes, and NCYM," JHEP 0303, 041 (2003), hep-th/0302049.

[14] M. Alishahiha and H. Yavartanoo, "Supergravity description of the large $N$ noncommutative dipole field theories," JHEP 0204, 031 (2002), hep-th/0202131; N. Sadooghi and M. Soroush, "Noncommutative dipole QED," Int. J. Mod. Phys. A 18, 97 (2003), hep-th/0206009. 
[15] O. J. Ganor and U. Varadarajan, "Nonlocal effects on D-branes in plane-wave backgrounds," JHEP 0211, 051 (2002), hep-th/0210035; M. Alishahiha and O. J. Ganor, "Twisted backgrounds, pp-waves and nonlocal field theories," JHEP 0303, 006 (2003), hep-th/0301080; M. Alishahiha and A. E. Mosaffa, "Semiclassical string solutions on deformed NS5-brane backgrounds and new pp-wave," hep-th/0302005.

[16] M. M. Sheikh-Jabbari, "Noncommutative super Yang-Mills theories with 8 supercharges and brane configurations," Nucl. Phys. B 587, 195 (2000), hep-th/0001089.

[17] M. Alishahiha, Y. Oz and M. M. Sheikh-Jabbari, "Supergravity and large N noncommutative field theories," JHEP 9911, 007 (1999), hep-th/9909215.

[18] A. M. Uranga, "Brane configurations for branes at conifolds, ” JHEP 9901, 022 (1999), hep-th/9811004.

[19] K. Dasgupta and S. Mukhi, "Brane constructions, conifolds and M-theory," Nucl. Phys. B 551, 204 (1999), hep-th/9811139.

[20] M. Bershadsky, C. Vafa and V. Sadov, "D-Strings on D-Manifolds," Nucl. Phys. B 463, 398 (1996), hep-th/9510225; H. Ooguri and C. Vafa, "Two-Dimensional Black Hole and Singularities of CY Manifolds," Nucl. Phys. B 463, 55 (1996), hepth/9511164.

[21] A. Hanany and A. M. Uranga, "Brane boxes and branes on singularities," JHEP 9805, 013 (1998), hep-th/9805139; M. Aganagic, A. Karch, D. Lust and A. Miemiec, "Mirror symmetries for brane configurations and branes at singularities," Nucl. Phys. B 569, 277 (2000), hep-th/9903093.

[22] J. D. Edelstein, K. Oh and R. Tatar, "Orientifold, geometric transition and large $N$ duality for SO/Sp gauge theories," JHEP 0105, 009 (2001), hep-th/0104037; K. Dasgupta, K. Oh and R. Tatar, "Geometric transition, large $N$ dualities and MQCD dynamics," Nucl. Phys. B 610, 331 (2001), hep-th/0105066; "Open/closed string dualities and Seiberg duality from geometric transitions in M-theory," JHEP 0208, 026 (2002), hep-th/0106040; K. Dasgupta, K. h. Oh, J. Park and R. Tatar, "Geometric transition versus cascading solution," JHEP 0201, 031 (2002), hep-th/0110050; A. Giveon, A. Kehagias and H. Partouche, "Geometric transitions, brane dynamics and gauge theories," JHEP 0112, 021 (2001), hep-th/0110115; K. h. Oh and R. Tatar, "Duality and confinement in $N=1$ supersymmetric theories from geometric transitions," Adv. Theor. Math. Phys. 6, 141 (2003), hep-th/0112040.

[23] R. de Mello Koch, K. Oh and R. Tatar, "Moduli space for conifolds as intersection of orthogonal D6 branes," Nucl. Phys. B 555, 457 (1999), hep-th/9812097.

[24] F. Arfaei and M.M. Sheikh-Jabbari, "Dirac Quantization of Open Strings and Noncommutativity in Branes," Nucl. Phys. B576 (2000) 578, hep-th/9906161; M.M. Sheikh-Jabbari and A. Shirzad, "Boundary Conditions as Dirac Constraints," hepth/9907055. 
[25] E. Witten, "Noncommutative Geometry and String Field Theory," Nucl. Phys. B268 (1986) 253.

[26] A. Connes, M.R. Douglas and A. Schwarz, "Noncommutative Geometry and Matrix Theory: Compactification on Tori," JHEP 9802 (1998) 003, hep-th/9711162.

[27] A. Sen, "Dynamics of multiple Kaluza-Klein monopoles in $M$ and string theory," Adv. Theor. Math. Phys. 1, 115 (1998), hep-th/9707042; "A note on enhanced gauge symmetries in $M$ - and string theory," JHEP 9709, 001 (1997), hep-th/9707123;

"Strong coupling dynamics of branes from M-theory," JHEP 9710, 002 (1997), hepth/9708002.

[28] R. Tatar, "A Note on Noncommutative Field Theory and Stability of Brane-Antibrane Systems," hep-th/0009213.

[29] L. Dolan and C. R. Nappi, "A scaling limit with many noncommutativity parameters," Phys. Lett. B 504, 329 (2001), hep-th/0009225.

[30] K. Dasgupta, C. Herdeiro, S. Hirano and R. Kallosh, "D3/D7 inflationary model and M-theory," Phys. Rev. D 65, 126002 (2002), hep-th/0203019.

[31] K. Dasgupta and S. Mukhi, "Brane Constructions, Fractional Branes and Anti- deSitter Domain Walls", JHEP 9907 (1999) 008, hep-th/9904131.

[32] D. s. Bak and N. Ohta, "Supersymmetric D2 anti-D2 strings," Phys. Lett. B 527, 131 (2002), hep-th/0112034.

[33] L. Cornalba and R. Schiappa, "Nonassociative star product deformations for D-brane worldvolumes in curved backgrounds," Commun. Math. Phys. 225, 33 (2002), hepth/0101219.

[34] D. Sadri and M. M. Sheikh-Jabbari, "String theory on parallelizable pp-waves," hepth/0304169.

[35] K. Becker, M. Becker, K. Dasgupta and P. S. Green, "Compactifications of heterotic theory on non-Kaehler complex manifolds. I," JHEP 0304, 007 (2003), hepth/0301161.

[36] E. Witten, "Ground ring of two-dimensional string theory," Nucl. Phys. B 373, 187 (1992), hep-th/9108004; E. Witten and B. Zwiebach, "Algebraic structures and differential geometry in 2-D string theory," Nucl. Phys. B 377, 55 (1992), hep-th/9201056.

[37] D. Ghoshal, D. P. Jatkar and S. Mukhi, "Kleinian singularities and the ground ring of $C=1$ string theory," Nucl. Phys. B 395, 144 (1993), hep-th/9206080.

[38] A. Hashimoto and S. Sethi, "Holography and string dynamics in time-dependent backgrounds," Phys. Rev. Lett. 89, 261601 (2002), hep-th/0208126. 